\title{
Boundary Control Problems for Oberbeck-Boussinesq Model of Heat and Mass Transfer
}

\author{
Gennady Alekseev ${ }^{1}$, Dmitry Tereshko ${ }^{1}$ and Vladislav Pukhnachev ${ }^{2}$ \\ ${ }^{1}$ Institute of Applied Mathematics of Far Eastern Branch RAS \\ ${ }^{2}$ Lavrentyev Institute of Hydrodynamics of Siberian Branch RAS \\ Russia
}

\section{Introduction}

Much attention has recently been given to statement and investigation of new problems for the models of heat and mass transfer. The control problems for Navier-Stokes and Oberbeck-Boussinesq equations are examples of such kind problems. This interest to control problems is connected with a variety of technical applications in science and engineering such as the crystal growth process, the aerodynamic drag reduction, the suppression of a turbulence and mass flow separation. In control problems the unknown densities of boundary or distributed sources, the coefficients of model differential equations or boundary conditions are recovered from minimum of certain cost functionals depending on controls and state (the solution to the original boundary value problem). The number of papers is devoted to study of control problems for models of heat and mass transfer. We mention in particular papers (Gunzburger et al., 1991; 1993; Ito \& Ravindran, 1998; Alekseev, 1998a;b; Alekseev \& Tereshko, 1998a;b; Capatina \& Stavre, 1998; Lee \& Imanuvilov, 2000a;b; Lee, 2003) devoted to theoretical study of control problems for stationary Oberbeck-Boussinesq equations.

Along with control problems, the inverse problems for models of heat and mass transfer play an important role. Importantly, these inverse problems can be reduced to corresponding control problems by choosing a suitable minimized cost functional that adequately describes the inverse problem in question (Alekseev, 2000; Alekseev \& Adomavichus, 2001; Alekseev, 2001; 2002; 2006; 2007a;b; Alekseev et al., 2008; Alekseev \& Soboleva, 2009; Alekseev \& Tereshko, 2010a). As a result, both control and inverse problems can be analyzed by applying a unified approach based on the constrained optimization theory in Hilbert or Banach spaces. These theoretical results, the increasing power of computers and the development of numerical methods for the flow simulation itself motivate the numerical study of the optimal flow control problems under consideration.

Oberbeck-Boussinesq equations are the most often used model of convection. Rigorous derivation of these equations from full model of viscous compressible heat conducting fluid can be found in (Gershuni \& Zhukhovitskii, 1976; Joseph, 1976). Limits of applicability of this model are indicated in (Pukhnachev, 1992) for heat convection description and in (Perera \& Sekerka, 1997) for concentration convection. In these papers new models of convection with non-solenoidal velocity field were proposed. In (Pukhnachev, 2004) the hierarchy of thermal gravitational convection models in closed domains, including the 
traditional Oberbeck-Boussinesq equations, was constructed using the asymptotic expansions of the original equations with respect to the parameters of weak compressibility and microconvection. This theory was further developed in (Andreev et al., 2008). It is shown there that the non-solenoidal effect results in a minor correction to the velocity field in the case of steady flows. At the same time, there are significant differences in predictions of classical and new models for dramatically unsteady flows, caused by large gradients of the initial temperature field or by long-term time-periodic changes in the boundary temperature regime. These differences are most noticeable in the case when the microconvection parameter $\eta=g l^{3} /\left(v \lambda_{1}\right)$ has the order one. Here $g$ is the acceleration of gravity, $l$ is the characteristic linear scale, $\lambda_{1}$ is the thermal diffusivity.

Let $\Omega$ be a bounded domain in the space $\mathbb{R}^{d}(d=2,3)$ with a Lipschitz boundary $\Gamma$ consisting of two parts $\Gamma_{D}$ and $\Gamma_{N}$. In this chapter we consider the model of heat and mass transfer in a viscous incompressible heat conducting fluid. The model consists of the Navier-Stokes equations and the convection-diffusion equations for the substance concentration and the temperature that are nonlinearly related via buoyancy in the Oberbeck-Boussinesq approximation and via convective mass and heat transfer. It is described by the equations

$$
\begin{gathered}
-v \Delta \mathbf{u}+(\mathbf{u} \cdot \nabla) \mathbf{u}+\nabla p=\mathbf{f}+\left(\beta_{C} C-\beta_{T} T\right) \mathbf{G}, \operatorname{div} \mathbf{u}=0 \text { in } \Omega, \mathbf{u}=\mathbf{g} \text { on } \Gamma, \\
-\lambda \Delta C+\mathbf{u} \cdot \nabla C+k C=f \text { in } \Omega, C=\psi \text { on } \Gamma_{D}, \lambda \partial C / \partial n=\chi \text { on } \Gamma_{N}, \\
-\lambda_{1} \Delta T+\mathbf{u} \cdot \nabla T=f_{1} \text { in } \Omega, T=\varphi \text { on } \Gamma_{D}, \lambda_{1}(\partial T / \partial n+\alpha T)=\eta \text { on } \Gamma_{N} .
\end{gathered}
$$

Here $\mathbf{u}, T$ and $C$ denote the velocity, temperature and substance concentration fields respectively, $p=P / \rho$ where $P$ is a pressure and $\rho=$ const is the density of medium, $v$ is the kinematic viscosity coefficient, $\mathbf{f}$ is the mass density of body forces, $\mathbf{G}$ is the gravitational acceleration vector, $k$ is the coefficient of decomposition of the substance due to chemical reactions, $f$ and $f_{1}$ are volume densities of the substance and heat sources, $\beta_{C}$ is the solutal expansion coefficient, $\beta_{T}$ is the volumetric thermal expansion coefficient, $\lambda$ and $\lambda_{1}$ are the diffusivity and thermal diffusivity coefficients, $\mathrm{g}$ is a given vector-function on $\Gamma, \psi$ and $\varphi$ are given functions on a part $\Gamma_{D}$ of $\Gamma, \chi$ and $\alpha, \eta$ are functions given on another part $\Gamma_{N}=\Gamma \backslash \Gamma_{D}$ of $\Gamma, \mathbf{n}$ is the unit outer normal. We note that all the quantities in (1)-(3) are dimensional and their dimensions are defined in terms of SI units.

Hereinafter it is assumed that the parameters $v, \lambda, \lambda_{1}, \beta_{C}, \beta_{T}, k$ are constants. This assumption is physically justified if the temperature $T$ varies in a small range (Batchelor, 2000). The temperature change has the greatest influence on the viscosity. For example the viscosity of water decreases by more than twice during heating from $1^{\circ} \mathrm{C}$ to $99^{\circ} \mathrm{C}$. As to the solvability of the boundary value problems for thermal convection model with temperature-dependent transport coefficients see (Goncharova, 2002) and references therein. Group-theoretical properties of the equations of this model and their exact solutions are considered in (Andreev et al., 1998). We note the anomalous property of water concerning with the nonmonotonic dependence of density on temperature near $T=4^{\circ} \mathrm{C}$. The dependence of parameters in equations (1)-(3) on the substance concentration $C$ is less significant. It should be noted also that the applicability of our concentration convection model is limited to small values of concentration $C$.

In the special case where $\beta_{T}=0$ and $k=0$ problem (1)-(3) splits into two: problem (1), (2) for the mass transfer model in the Oberbeck-Boussinesq approximation (see (Joseph, 1976)), and the linear boundary value problem (3) (for given $\mathbf{u}$ ) for temperature $T$, which describes 
the convection-diffusion of a heat. We shall refer to problem (1), (2) at $\beta_{T}=0$ as Model 1. In another special case where $\beta_{C}=0$ problem (1)-(3) splits into problem (1), (3) for a heat transfer model in the Oberbeck-Boussinesq approximation (see (Joseph, 1976)), and the linear boundary value problem (2) for substance concentration $C$. We shall refer to problem (1), (3) at $\beta_{C}=0$ as Model 2. Finally problem (1)-(3) itself is referred to as Model 3.

Our goal is the study of the boundary control problems for the models under consideration. The problems consist in minimization of certain cost functionals depending on the state and controls. In order to formulate a boundary control problem for Model 1 we divide the set of all data of Model 1 into two groups: the group of controls containing the functions $\mathbf{g} \in \mathbf{H}^{1 / 2}(\Gamma)$ and $\chi \in L^{2}\left(\Gamma_{N}\right)$, which play the role of controls, and the group of fixed data comprising the invariable functions $\mathbf{f}, f, k, \beta_{C}$ and $\psi$. We assume that the controls $\mathbf{g}$ and $\chi$ vary in some closed convex sets $K_{1} \subset \mathbf{H}^{1 / 2}(\Gamma)$ and $K_{2} \subset L^{2}\left(\Gamma_{N}\right)$. The mathematical statement of the boundary control problem for Model 1 is as follows: to find a pair $(\mathbf{x}, u)$, where $u=(\mathbf{g}, \chi) \in K=K_{1} \times K_{2}$, $\mathbf{x}=(\mathbf{u}, p, C) \in X=\mathbf{H}^{1}(\Omega) \times L_{0}^{2}(\Omega) \times H^{1}(\Omega)$ such that $F(\mathbf{x}, u) \equiv 0$ and

$$
J(\mathbf{x}, \mathbf{g}, \chi) \equiv \frac{\mu_{0}}{2} I(\mathbf{x})+\frac{\mu_{1}}{2}\|\mathbf{g}\|_{\mathbf{H}^{1 / 2}(\Gamma)}^{2}+\frac{\mu_{2}}{2}\|\chi\|_{L^{2}\left(\Gamma_{N}\right)}^{2} \rightarrow \text { inf. }
$$

Here $F(\mathbf{x}, u)=0$ is the operator constraint in the form of a weak formulation of Model $1 ; \mu_{0}$, $\mu_{1}, \mu_{2}$ are nonnegative constants, $I(\mathbf{x})$ is a cost functional.

Similar boundary control problems can be formulated for Models 2 and 3 . We divide the set of all data of Model 2 into two groups: the group of controls containing the functions $\mathbf{g} \in \mathbf{H}^{1 / 2}(\Gamma)$ and $\eta \in L^{2}\left(\Gamma_{N}\right)$ and the group of fixed data comprising the invariable functions $\mathbf{f}, f_{1}, \beta_{T}, \alpha$ and $\varphi$. It is assumed that the controls $\mathbf{g}$ and $\eta$ vary in some closed convex sets $K_{1} \subset \mathbf{H}^{1 / 2}(\Gamma)$ and $K_{3} \subset L^{2}\left(\Gamma_{N}\right)$. A boundary control problem for Model 2 consists of finding a pair $(\mathbf{x}, u)$, where $u=(\mathbf{g}, \eta) \in K=K_{1} \times K_{3}, \mathbf{x}=(\mathbf{u}, p, T) \in X=\mathbf{H}^{1}(\Omega) \times L_{0}^{2}(\Omega) \times H^{1}(\Omega)$ such that $G(\mathbf{x}, u)=0$ and

$$
J(\mathbf{x}, \mathbf{g}, \eta) \equiv \frac{\mu_{0}}{2} I(\mathbf{x})+\frac{\mu_{1}}{2}\|\mathbf{g}\|_{\mathbf{H}^{1 / 2}(\Gamma)}^{2}+\frac{\mu_{3}}{2}\|\eta\|_{L^{2}\left(\Gamma_{N}\right)}^{2} \rightarrow \text { inf. }
$$

Here $G(\mathbf{x}, u)=0$ is the operator constraint in the form of a weak formulation of Model 2, $\mu_{0}, \mu_{1}$ and $\mu_{3}$ are nonnegative constants. Finally, a boundary control problem for Model 3 consists of finding a pair $(\mathbf{x}, u)$, where $u=(\mathbf{g}, \chi, \eta) \in K=K_{1} \times K_{2} \times K_{3}, \mathbf{x}=(\mathbf{u}, p, C, T) \in X=$ $\mathbf{H}^{1}(\Omega) \times L_{0}^{2}(\Omega) \times H^{1}(\Omega) \times H^{1}(\Omega)$ such that $H(\mathbf{x}, u)=0$ and

$$
J(\mathbf{x}, \mathbf{g}, \chi, \eta) \equiv \frac{\mu_{0}}{2} I(\mathbf{x})+\frac{\mu_{1}}{2}\|\mathbf{g}\|_{\mathbf{H}^{1 / 2}(\Gamma)}^{2}+\frac{\mu_{2}}{2}\|\chi\|_{L^{2}\left(\Gamma_{N}\right)}^{2}+\frac{\mu_{3}}{2}\|\eta\|_{L^{2}\left(\Gamma_{N}\right)}^{2} \rightarrow \text { inf. }
$$

Here $H(\mathbf{x}, u)=0$ is the operator constraint in the form of a weak formulation of Model 3.

The work consists of two parts. In the first part the solvability theorems for boundary control problems under study are formulated and proved. Optimality systems describing the first-order necessary optimality conditions are derived and analyzed. Sufficient conditions to the data ensuring the local uniqueness and stability of optimal solutions for concrete tracking-type functionals are established. The local uniqueness conditions are rather cumbersome. To simplify them, we introduce analogues of dimensionless parameters widely used in fluid dynamics, namely, the Reynolds number and the diffusion or temperature Rayleigh and Prandtl numbers. In terms of these parameters, the uniqueness conditions can be written in a relatively simple form and are similar to those for the coefficient inverse problems for the stationary linear convection-diffusion-reaction equation (see e.g. (Alekseev \& Tereshko, 2008)). 
In the second part a numerical algorithm based on Newton's method for the optimality system and finite element method for linearized boundary value problems is formulated and analyzed. Some computational results connected with the vortex reduction in the steady $2 \mathrm{D}$ viscous fluid flow around a cylinder in a channel by means of the temperature and hydrodynamic controls on some parts of the boundary are given and discussed. The details of theoretical and numerical studies can be found in (Alekseev \& Tereshko, 2008).

\section{Statement of boundary control problem for mass transfer model}

We begin our study with consideration of Model 1 having in denotions of Sect. 1 the form

$$
\begin{aligned}
& -v \Delta \mathbf{u}+(\mathbf{u} \cdot \nabla) \mathbf{u}+\nabla p=\mathbf{f}+\beta_{C} C \mathbf{G}, \operatorname{div} \mathbf{u}=0 \text { in } \Omega, \mathbf{u}=\mathbf{g} \text { on } \Gamma, \\
& -\lambda \Delta C+\mathbf{u} \cdot \nabla C+k C=f \text { in } \Omega, C=\psi \text { on } \Gamma_{D}, \lambda \partial C / \partial n=\chi \text { on } \Gamma_{N} .
\end{aligned}
$$

Under theoretical study of control problems for Model 1 we shall use the Sobolev spaces $H^{s}(D)$ with $s \in \mathbb{R}$ and the spaces $L^{r}(D)$ with $r \geq 2$ where $D$ denotes $\Omega$, its subset $Q, \Gamma$ or its part $\Gamma_{0}$ with positive measure. The corresponding spaces of vector functions are denoted by $\mathbf{H}^{s}(D)$ and $\mathbf{L}^{r}(D)$. The norms and inner products in $H^{s}(Q), H^{s}(\Gamma)$ and in their vector analogies are denoted by $\|\cdot\|_{s, Q},\|\cdot\|_{s, \Gamma}$ and $(\cdot, \cdot)_{s, Q},(\cdot, \cdot)_{s, \Gamma}$. The inner products and norms in $L^{2}(Q)$ or in $L^{2}(Q)$ are denoted by $(\cdot, \cdot)_{Q}$ and $\|\cdot\|_{Q}$. If $Q=\Omega$ then we set $\|\cdot\|_{\Omega}=\|\cdot\|$, $(\cdot, \cdot)_{\Omega}=(\cdot, \cdot)$. The inner product and norm in $L^{2}\left(\Gamma_{N}\right)$ are denoted by $(\cdot, \cdot)_{\Gamma_{N}}$ and $\|\cdot\|_{\Gamma_{N}}$. The norm and seminorm in $H^{1}(\Omega)$ or in $\mathbf{H}^{1}(\Omega)$ are denoted by $\|\cdot\|_{1}$ and $|\cdot|_{1}$. The duality relation for the pair of dual spaces $X$ and $X^{*}$ is denoted by $\langle\cdot, \cdot\rangle_{X^{*} \times X}$ or simply $\langle\cdot, \cdot\rangle$. Let the following assumptions hold:

(i) $\Omega$ is a bounded domain in $\mathbb{R}^{d}, d=2,3$ with a boundary $\Gamma \in C^{0,1}$ consisting of $N$ connected components $\Gamma_{i}, i=1,2, \ldots, N$. The open segments $\Gamma_{D}$ and $\Gamma_{N}$ of $\Gamma$ obey the conditions $\Gamma_{D} \in C^{0,1}$, $\Gamma_{N} \in C^{0,1}, \Gamma_{D} \neq \varnothing, \Gamma_{D} \cap \Gamma_{N}=\varnothing, \Gamma=\bar{\Gamma}_{D} \cup \bar{\Gamma}_{N}$.

Let $\mathcal{D}(\Omega)$ be the space of infinitely differentiable finite in $\Omega$ functions, $H_{0}^{1}(\Omega)$ be a closure of $\mathcal{D}(\Omega)$ in $H^{1}(\Omega), \mathbf{H}_{0}^{1}(\Omega)=H_{0}^{1}(\Omega)^{d}, \mathbf{V}=\left\{\mathbf{v} \in \mathbf{H}_{0}^{1}(\Omega): \operatorname{div} \mathbf{v}=0\right\}, \mathbf{H}^{-1}(\Omega)=\mathbf{H}_{0}^{1}(\Omega)^{*}, L_{0}^{2}(\Omega)=$ $\left\{p \in L^{2}(\Omega):(p, 1)=0\right\}, \mathcal{T}=H^{1}\left(\Omega, \Gamma_{D}\right) \equiv\left\{S \in H^{1}(\Omega):\left.S\right|_{\Gamma_{D}}=0\right\}, L_{+}^{2}(D)=\left\{u \in L^{2}(D): u \geq\right.$ $0\}$. We shall use the following inequalities which are implied by the embedding theorems and the continuity of the trace operator:

$$
\|\mathbf{u}\|_{Q} \leq c_{Q}\|\mathbf{u}\|_{1},\|\operatorname{rot} \mathbf{u}\| \leq c_{r}\|\mathbf{u}\|_{1},\|\mathbf{u}\|_{1 / 2, \Gamma} \leq c_{\Gamma}\|\mathbf{u}\|_{1} \forall \mathbf{u} \in \mathbf{H}^{1}(\Omega) .
$$

Here $c_{Q}, c_{r}, c_{\Gamma}$ are constants depending on $\Omega$.

Together with $\mathbf{H}^{1}(\Omega)$ and $\mathbf{H}^{1 / 2}(\Gamma)$ we shall consider their closed subspaces $\tilde{\mathbf{H}}^{1}(\Omega)=$ $\left\{\mathbf{u} \in \mathbf{H}^{1}(\Omega):\left.\mathbf{u} \cdot \mathbf{n}\right|_{\Gamma_{N}}=0,(\mathbf{u}, \mathbf{n})_{\Gamma_{i}}=0, i=\overline{1, N}\right\}, \mathbf{H}_{\operatorname{div}}^{1}(\Omega)=\left\{\mathbf{v} \in \mathbf{H}^{1}(\Omega): \operatorname{div} \mathbf{v}=0\right\}$, $\tilde{\mathbf{H}}_{\text {div }}^{1}(\Omega)=\left\{\mathbf{v} \in \tilde{\mathbf{H}}^{1}(\Omega): \operatorname{div} \mathbf{v}=0\right\}, \tilde{\mathbf{H}}^{1 / 2}(\Gamma)=\left\{\left.\mathbf{u}\right|_{\Gamma}: \mathbf{u} \in \tilde{\mathbf{H}}^{1}(\Omega)\right\}$, and also duals $\tilde{\mathbf{H}}^{1}(\Omega)^{*}$, $\tilde{\mathbf{H}}^{1 / 2}(\Gamma)^{*}$ of the spaces $\tilde{\mathbf{H}}^{1}(\Omega), \tilde{\mathbf{H}}^{1 / 2}(\Gamma)$. Let us introduce the following bilinear and trilinear forms: $a_{0}: \mathbf{H}^{1}(\Omega)^{2} \rightarrow \mathbb{R}, b: \mathbf{H}^{1}(\Omega) \times L_{0}^{2}(\Omega) \rightarrow \mathbb{R}, a_{1}: H^{1}(\Omega)^{2} \rightarrow \mathbb{R}, b_{1}: H^{1}(\Omega) \times \mathbf{H}_{0}^{1}(\Omega) \rightarrow \mathbb{R}$, $c: \mathbf{H}^{1}(\Omega)^{3} \rightarrow \mathbb{R}, c_{1}: \mathbf{H}^{1}(\Omega) \times H^{1}(\Omega) \times H^{1}(\Omega) \rightarrow \mathbb{R}$ by

$$
\begin{gathered}
a_{0}(\mathbf{u}, \mathbf{v})=(\nabla \mathbf{u}, \nabla \mathbf{v}), b(\mathbf{v}, q)=-(\operatorname{div} \mathbf{v}, q), c(\mathbf{u}, \mathbf{v}, \mathbf{w})=((\mathbf{u} \cdot \operatorname{grad}) \mathbf{v}, \mathbf{w}), \\
a_{1}(C, S)=(\nabla C, \nabla S), b_{1}(S, \mathbf{v})=(\mathbf{b} S, \mathbf{v}), c_{1}(\mathbf{u}, C, S)=(\mathbf{u} \cdot \nabla C, S), \mathbf{b} \equiv \beta_{C} \mathbf{G} .
\end{gathered}
$$


We note that forms $c$ and $c_{1}$ possess the next properties (Alekseev \& Tereshko, 2008; Girault \& Raviart, 1986):

$$
\begin{gathered}
c(\mathbf{u}, \mathbf{v}, \mathbf{w})=-c(\mathbf{u}, \mathbf{w}, \mathbf{v}), c(\mathbf{u}, \mathbf{v}, \mathbf{v})=0 \forall \mathbf{u} \in \mathbf{H}_{\operatorname{div}}^{1}(\Omega),(\mathbf{v}, \mathbf{w}) \in \mathbf{H}_{0}^{1}(\Omega) \times \mathbf{H}^{1}(\Omega), \\
c_{1}(\mathbf{u}, C, S)=-c_{1}(\mathbf{u}, S, C), c_{1}(\mathbf{u}, C, C)=0 \forall \mathbf{u} \in \tilde{\mathbf{H}}_{\operatorname{div}}^{1}(\Omega),(C, S) \in \mathcal{T} \times H^{1}(\Omega) .
\end{gathered}
$$

Besides all the forms are continuous and the following technical lemma holds (Alekseev \& Tereshko, 2008).

Lemma 1. Under conditions (i) there exist constants $\delta_{0}, \delta_{1}, \gamma_{0}, \gamma_{1}, \gamma_{2}$ and $\beta_{1}$ depending on $\Omega$ such that

$$
\begin{gathered}
\left|a_{0}(\mathbf{u}, \mathbf{v})\right| \leq\|\mathbf{u}\|_{1}\|\mathbf{v}\|_{1} \forall(\mathbf{u}, \mathbf{v}) \in \mathbf{H}^{1}(\Omega)^{2}, a_{0}(\mathbf{v}, \mathbf{v}) \geq \delta_{0}\|\mathbf{v}\|_{1}^{2} \forall \mathbf{v} \in \mathbf{H}_{0}^{1}(\Omega), \\
\left|a_{1}(C, S)\right| \leq\|C\|_{1}\|S\|_{1} \forall(C, S) \in H^{1}(\Omega) \times \in H^{1}(\Omega), a_{1}(C, C) \geq \delta_{1}\|C\|_{1}^{2} \forall C \in \mathcal{T}, \\
|c(\mathbf{u}, \mathbf{v}, \mathbf{w})| \leq \gamma_{0}\|\mathbf{u}\|_{1}\|\mathbf{v}\|_{1}\|\mathbf{w}\|_{1} \forall(\mathbf{u}, \mathbf{v}, \mathbf{w}) \in \mathbf{H}^{1}(\Omega)^{3}, \\
\left|c_{1}(\mathbf{u}, C, S)\right| \leq \gamma_{1}\|\mathbf{u}\|_{1}\|C\|_{1}\|S\|_{1} \forall \mathbf{u} \in \mathbf{H}^{1}(\Omega),(C, S) \in H^{1}(\Omega) \times \in H^{1}(\Omega), \\
\left|b_{1}(C, \mathbf{v})\right| \leq \beta_{1}\|C\|_{1}\|\mathbf{v}\|_{1} \forall C \in H^{1}(\Omega), \mathbf{v} \in \mathbf{H}_{0}^{1}(\Omega), \\
\left|(\chi, C)_{\Gamma_{N}}\right| \leq\|\chi\|_{\Gamma_{N}}\|C\|_{\Gamma_{N}} \leq \gamma_{2}\|\chi\|_{\Gamma_{N}}\|C\|_{1} \forall C \in H^{1}(\Omega) .
\end{gathered}
$$

Bilinear form $b(\cdot, \cdot)$ satisfies inf-sup condition

$$
\inf _{q \in L_{0}^{2}(\Omega), q \neq 0} \sup _{\mathbf{v} \in \mathbf{H}_{0}^{1}(\Omega), \mathbf{v} \neq 0} \frac{b(\mathbf{v}, q)}{\|\mathbf{v}\|_{1}\|q\|} \geq \beta=\text { const }>0 .
$$

Let in addition to (i) the following conditions take place:

(ii) $\mathbf{f} \in \mathbf{H}^{-1}(\Omega), \mathbf{b} \in \mathbf{L}^{2}(\Omega), f \in L^{2}(\Omega), k \in L_{+}^{2}(\Omega), \psi \in H^{1 / 2}\left(\Gamma_{D}\right)$;

(iii) $\mathbf{g} \in \tilde{\mathbf{H}}^{1 / 2}(\Gamma), \chi \in L^{2}\left(\Gamma_{N}\right)$.

In order to formulate an extremum problem for Model 1 we divide the set of all input data in problem (5), (6) into two groups. One consists of control functions $\mathbf{g}$ and $\chi$, and the other consists of fixed data, namely $\mathbf{f}, \mathbf{b}, f, k$ and $\psi$. Assume that controls $\mathbf{g}, \chi$ vary over some sets $K_{1}$ and $K_{2}$ such that

(j) $K_{1} \subset \tilde{\mathbf{H}}^{1 / 2}(\Gamma), K_{2} \subset L^{2}\left(\Gamma_{N}\right)$ are nonempty convex closed subsets.

Let $X=\tilde{\mathbf{H}}^{1}(\Omega) \times L_{0}^{2}(\Omega) \times H^{1}(\Omega), Y=\mathbf{H}^{-1}(\Omega) \times L_{0}^{2}(\Omega) \times \tilde{\mathbf{H}}^{1 / 2}(\Gamma) \times \mathcal{T}^{*} \times H^{1 / 2}\left(\Gamma_{D}\right), \mathbf{x}=$ $(\mathbf{u}, p, C) \in X$. Introduce an operator $F \equiv\left(F_{1}, F_{2}, F_{3}, F_{4}, F_{5}\right): X \times K_{1} \times K_{2} \rightarrow Y$, defined by

$$
\begin{gathered}
\left\langle F_{1}(\mathbf{x}, u), \mathbf{v}\right\rangle=v a_{0}(\mathbf{u}, \mathbf{v})+c(\mathbf{u}, \mathbf{u}, \mathbf{v})+b(\mathbf{v}, p)-b_{1}(C, \mathbf{v})-\langle\mathbf{f}, \mathbf{v}\rangle, \\
\left\langle F_{2}(\mathbf{x}, u), r\right\rangle=b(\mathbf{u}, r) \equiv-(\operatorname{div} \mathbf{u}, r), F_{3}(\mathbf{x}, u)=\left.\mathbf{u}\right|_{\Gamma}-\mathbf{g}, F_{5}(\mathbf{x}, u)=\left.C\right|_{\Gamma_{D}}-\psi, \\
\left\langle F_{4}(\mathbf{x}, u), S\right\rangle=\lambda a_{1}(C, S)+(k C, S)+c_{1}(\mathbf{u}, C, S)-(f, S)-(\chi, S)_{\Gamma_{N}} .
\end{gathered}
$$

We multiply the first equation in (5) by $\mathbf{v} \in \mathbf{H}_{0}^{1}(\Omega)$, the equation in (6) by $S \in \mathcal{T}$, integrate the results over $\Omega$ with use of Green formulas, and use boundary conditions in (5), (6) to obtain a 
weak formulation of problem 1 . It consists of finding a triple $\mathbf{x}=(\mathbf{u}, p, C) \in X$ satisfying the relations

$$
\begin{gathered}
v a_{0}(\mathbf{u}, \mathbf{v})+c(\mathbf{u}, \mathbf{u}, \mathbf{v})+b(\mathbf{v}, p)-b_{1}(C, \mathbf{v})=\langle\mathbf{f}, \mathbf{v}\rangle \forall \mathbf{v} \in \mathbf{H}_{0}^{1}(\Omega), \\
\lambda a_{1}(C, S)+(k C, S)+c_{1}(\mathbf{u}, C, S)=\langle l, S\rangle \equiv(f, S)+(\chi, S)_{\Gamma_{N}} \forall S \in \mathcal{T}, \\
\operatorname{div} \mathbf{u}=0 \text { in } \Omega,\left.\mathbf{u}\right|_{\Gamma}=\mathbf{g},\left.C\right|_{\Gamma_{D}}=\psi,
\end{gathered}
$$

which one can rewrite in an equivalent form of the operator equation

$$
F(\mathbf{x}, u) \equiv F(\mathbf{u}, p, C, \mathbf{g}, \chi)=0 .
$$

This triple $(\mathbf{u}, p, C) \in X$ will be called the weak solution to problem (5), (6).

Let $I: X \rightarrow \mathbb{R}$ be a weakly lower semicontinuous cost functional. Setting $K=K_{1} \times K_{2}, u=$ $(\mathbf{g}, \chi), u_{0}=(\mathbf{f}, \mathbf{b}, f, k, \psi)$ we formulate the following constrained minimization problem

$$
J(\mathbf{x}, u)=\left(\mu_{0} / 2\right) I(\mathbf{x})+\left(\mu_{1} / 2\right)\|\mathbf{g}\|_{1 / 2, \Gamma}^{2}+\left(\mu_{2} / 2\right)\|\chi\|_{\Gamma_{N}}^{2} \rightarrow \inf , F(\mathbf{x}, u)=0,(\mathbf{x}, u) \in X \times K .
$$

Here $\mu_{0}>0$ and $\mu_{1} \geq 0, \mu_{2} \geq 0$ are positive dimensional parameters which serve to regulate the relative importance of each of the terms in (21). Another purpose of introducing $\mu_{l}$ is to ensure the uniqueness and stability of solutions to control problems under consideration (see below). The possible cost functionals are defined as

$$
I_{1}(\mathbf{x})=\left\|\mathbf{v}-\mathbf{v}_{d}\right\|_{Q}^{2}, I_{2}(\mathbf{x})=\left\|\mathbf{v}-\mathbf{v}_{d}\right\|_{1, Q}^{2}, I_{3}(\mathbf{x})=\left\|\operatorname{rot} \mathbf{v}-\eta_{d}\right\|_{Q^{\prime}}^{2} I_{4}(x)=\left\|p-p_{d}\right\|_{Q}^{2} .
$$

Here $Q$ is a subset of $\Omega, \mathbf{v}_{d} \in \mathbf{L}^{2}(Q)$ ( or $\mathbf{v}_{d} \in \mathbf{H}^{1}(Q)$ ), $\eta_{d} \in \mathbf{L}^{2}(Q)$ and $p_{d} \in L^{2}(Q)$ are functions which are interpreted as measured velocity, vorticity or pressure fields. Define $Z_{a d}=\{(\mathbf{x}, u) \in$ $X \times K: F(\mathbf{x}, u)=0, J(\mathbf{x}, u)<\infty\}$. Let us assume in addition to (j) that

(j) $\mu_{0}>0, \mu_{1} \geq 0, \mu_{2} \geq 0$ and $K$ is a bounded subset or $\mu_{0}>0, \mu_{1}>0, \mu_{2}>0$ and functional $I$ is bounded from below.

According to general theory of extremum problems (see (Ioffe \& Tikhomirov, 1979)) we introduce an element $\mathbf{y}^{*}=\left(\xi, \sigma, \zeta, \theta, \zeta^{c}\right) \in Y^{*}=\mathbf{H}_{0}^{1}(\Omega) \times L_{0}^{2}(\Omega) \times \tilde{\mathbf{H}}^{1 / 2}(\Gamma)^{*} \times \mathcal{T} \times H^{1 / 2}\left(\Gamma_{D}\right)^{*}$ which is reffered to as the adjoint state and define the Lagrangian $\mathcal{L}: X \times K \times \mathbb{R}^{+} \times Y^{*} \rightarrow \mathbb{R}$, where $\mathbb{R}^{+}=\{\lambda \in \mathbb{R}: \lambda \geq 0\}$, by the formula

$$
\begin{gathered}
\mathcal{L}\left(\mathbf{x}, u, \lambda_{0}, \mathbf{y}^{*}\right)=\lambda_{0} J(\mathbf{x}, u)+\left\langle F_{1}(\mathbf{x}, u), \xi\right\rangle+\left(F_{2}(\mathbf{x}, u), q\right)+ \\
\left\langle\zeta, F_{3}(\mathbf{x}, u)\right\rangle_{\Gamma}+\varkappa\left\langle F_{4}(\mathbf{x}, u), \theta\right\rangle+\varkappa\left\langle\zeta^{c}, F_{5}(\mathbf{x}, u)\right\rangle_{\Gamma_{D}} .
\end{gathered}
$$

Here $\langle\zeta, \mathbf{g}\rangle_{\Gamma}=\langle\zeta, \mathbf{g}\rangle_{\tilde{\mathbf{H}}^{1 / 2}(\Gamma)^{*} \times \tilde{\mathbf{H}}^{1 / 2}(\Gamma)}$ for $\zeta \in \tilde{\mathbf{H}}^{1 / 2}(\Gamma)^{*},\left\langle\zeta^{\mathcal{C}}, \psi\right\rangle_{\Gamma_{D}}=\left\langle\zeta^{\mathcal{C}}, \psi\right\rangle_{H^{1 / 2}\left(\Gamma_{D}\right)^{*} \times H^{1 / 2}\left(\Gamma_{D}\right)}$ for $\zeta^{\mathcal{C}} \in H^{1 / 2}\left(\Gamma_{D}\right)^{*}, \varkappa$ is a dimensional parameter. Let the dimension $[\varkappa]$ be chosen so that the dimensions of $\xi, s, \theta$ at the adjoint state $\mathbf{y}^{*}$ coincide with those at the basic state $\mathbf{x}=(\mathbf{u}, p, C)$ i.e.

$$
[\xi]=[\mathbf{u}]=L_{0} T_{0}^{-1},[\theta]=[C]=M_{0} L_{0}^{-3},[s]=[p]=L_{0}^{2} T_{0}^{-2} .
$$

Here $L_{0}, T_{0}, M_{0}$ denote the SI dimensions of the length, time and mass units expressed in meters, seconds and kilograms respectively. A simple analysis of (23) shows that necessary condition for fulfillment of (23) is $[\varkappa]=L_{0}^{8} T_{0}^{-2} M_{0}^{-2}$ (see (Alekseev \& Tereshko, 2008)).

Below we shall use some results concerning problem (5), (6) and extremum problem (21). The proofs of the theorems are simular to those in (Alekseev \& Tereshko, 2008). 
Theorem 1. Let conditions (i), (ii) be satisfied. Then for any $u \in K$ problem (5), (6) has a weak solution $(\mathbf{u}, p, C) \in X$ that satisfies the estimates $\|\mathbf{u}\|_{1} \leq M_{\mathbf{u}}\left(u_{0}, u\right),\|p\| \leq M_{p}\left(u_{0}, u\right),\|C\|_{1} \leq$ $M_{C}\left(u_{0}, u\right)$. Here $M_{\mathbf{u}}\left(u_{0}, u\right), M_{p}\left(u_{0}, u\right)$ and $M_{C}\left(u_{0}, u\right)$ are nondecreasing continuous functions of the norms $\|\mathbf{f}\|_{-1},\|\mathbf{b}\|,\|f\|,\|k\|_{,}\|\psi\|_{1 / 2, \Gamma_{D}},\|\mathbf{g}\|_{1 / 2, \Gamma},\|\chi\|_{\Gamma_{N}}$. If the functions $\mathbf{f}, \mathbf{b}, f, k, \psi, \mathbf{g}, \chi$ are small (or the viscosity $v$ is high) in the sense that

$$
\frac{\gamma_{0} M_{\mathbf{u}}\left(u_{0}, u\right)}{\delta_{0} v}+\frac{1}{\delta_{0} v} \frac{\beta_{1} \gamma_{1} M_{C}^{0}\left(u_{0}, u\right)}{\delta_{1} \lambda}<1,
$$

then the weak solution to problem 1 is unique. Here $\delta_{0}, \delta_{1}, \gamma_{0}, \gamma_{1}, \beta_{1}$ are the constants from (10)-(14).

Theorem 2. Under conditions ( $i)$, (ii), ( $j)$ and (jj) let $I: X \rightarrow \mathbb{R}$ be a weakly lower semicontinuous functional and $Z_{a d} \neq \varnothing$. Then control problem (21) has at least one solution.

Theorem 3. Under conditions (i), (ii), (j) let $\mu_{0}>0, \mu_{l}>0$ or $\mu_{0}>0, \mu_{l} \geq 0$ and $K_{l}$ be the bounded sets, $l=1,2$. Then control problem (21) has at least one solution for $I=I_{k}, \bar{k}=1,2,3,4$.

Theorem 4. Under conditions (i), (ii), (j) and (jj) let $(\hat{\mathbf{x}}, \hat{u}) \equiv(\hat{\mathbf{u}}, \hat{p}, \hat{C}, \hat{\mathbf{g}}, \hat{\chi}) \in X \times K$ be a local minimizer in problem (21) and let the functional I be continuously differentiable at the point $\hat{\mathbf{x}}$. Then, there exists a nonzero Lagrange multiplier $\left(\lambda_{0}, \mathbf{y}^{*}\right)=\left(\lambda_{0}, \xi, \sigma, \zeta, \theta, \zeta^{c}\right) \in \mathbb{R}^{+} \times \mathbf{H}_{0}^{1}(\Omega) \times L_{0}^{2}(\Omega) \times$ $\tilde{\mathbf{H}}^{1 / 2}(\Gamma)^{*} \times \mathcal{T} \times H^{1 / 2}\left(\Gamma_{D}\right)^{*}$ that satisfies the Euler-Lagrange equation $F_{\mathbf{x}}^{\prime}(\hat{\mathbf{x}}, \hat{u})^{*} \mathbf{y}^{*}=-\lambda_{0} J_{\mathbf{x}}^{\prime}(\hat{\mathbf{x}}, \hat{u})$, which is equivalent to the identities

$$
\begin{gathered}
v a_{0}(\mathbf{w}, \xi)+c(\hat{\mathbf{u}}, \mathbf{w}, \xi)+c(\mathbf{w}, \hat{\mathbf{u}}, \xi)+\varkappa c_{1}(\mathbf{u}, \hat{C}, \theta)+b(\mathbf{w}, \sigma)+\langle\zeta, \mathbf{w}\rangle_{\Gamma}= \\
-\lambda_{0}\left(\mu_{0} / 2\right)\left\langle I_{\mathbf{u}}^{\prime}(\hat{\mathbf{x}}), \mathbf{w}\right\rangle \forall \mathbf{w} \in \tilde{\mathbf{H}}^{1}(\Omega), b(\xi, r) \equiv-(\operatorname{div} \xi, r)=-\lambda_{0}\left(\mu_{0} / 2\right)\left(I_{p}^{\prime}(\hat{\mathbf{x}}), r\right) \forall r \in L_{0}^{2}(\Omega), \\
\varkappa\left[\lambda a_{1}(\tau, \theta)+(k \tau, \theta)+c_{1}(\hat{\mathbf{u}}, \tau, \theta)+\left\langle\zeta^{c}, \tau\right\rangle_{\Gamma_{D}}\right]-b_{1}(\tau, \xi)= \\
-\lambda_{0}\left(\mu_{0} / 2\right)\left\langle I_{C}^{\prime}(\hat{\mathbf{x}}), \tau\right\rangle \forall \tau \in H^{1}(\Omega),
\end{gathered}
$$

and satisfies the minimum principle $\mathcal{L}\left(\hat{\mathbf{x}}, \hat{u}, \lambda_{0}, \mathbf{y}^{*}\right) \leq \mathcal{L}\left(\hat{\mathbf{x}}, u, \lambda_{0}, \mathbf{y}^{*}\right)$ for all $u \in K$ and the variational inequality

$$
\lambda_{0} \mu_{1}(\hat{\mathbf{g}}, \mathbf{g}-\hat{\mathbf{g}})_{1 / 2, \Gamma}-\langle\zeta, \mathbf{g}-\hat{\mathbf{g}}\rangle_{\Gamma}+\lambda_{0} \mu_{2}(\hat{\chi}, \chi-\hat{\chi})_{\Gamma_{N}}-\varkappa\langle\theta, \chi-\hat{\chi}\rangle_{\Gamma_{N}} \geq 0 \forall u=(\mathbf{g}, \chi)
$$

Theorem 5. Let the assumptions of Theorem 4 be satisfied and inequality (24) hold for all $u \in K$. Then: 1) homogeneous problem (25), (26) (if $\lambda_{0}=0$ ) has only trivial solution $\mathbf{y}^{*} \equiv\left(\xi, \sigma, \zeta, \theta, \zeta^{\mathcal{c}}\right)=0$; 2) any nontrivial Lagrange multiplier satisfying (25), (26) is regular, i.e. it has the form $\left(1, \mathbf{y}^{*}\right)$. Relations (25), (26), together with variational inequality (27) and operator constraint (20) constitute an optimality system. It consists of three parts. The first part has the form of a weak formulation (17)-(19) of problem (5), (6), which is equivalent to operator equation (20). The second part consists of identities (25), (26) for the Lagrange multipliers $\xi, \sigma, \zeta, \theta$ and $\zeta^{c}$. Finally, the last part of the optimality system is the variational inequality (27) with respect to controls $\mathbf{g}$ and $\chi$ which is the consequence of the minimum principle.

Remark 1. We emphasize that the multiplier (adjoint velocity) $\xi$ is in a general case a nonsolenoidal vector-function except the situation when the cost functional $I$ independent of pressure $p$. Only in this case it follows from (25) that $\operatorname{div} \xi=0$ and moreover $\xi \in \mathbf{V}$. 
Remark 2. Denote by $\mathbf{g}_{i}=\left.\mathbf{g}\right|_{\Gamma_{i}}$ the restriction of the boundary vector $\mathbf{g}$ to the component $\Gamma_{i}$ of $\Gamma$ and introduce values (flows) $q_{i}$ of the vector $\mathbf{g}_{i}$ through $\Gamma_{i}$ by $q_{i}=\left(\mathbf{g}_{i}, \mathbf{n}\right)_{\Gamma_{i}} \equiv \int_{\Gamma_{i}} \mathbf{g} \cdot \mathbf{n} d \sigma$. We note that the incompressibility condition $\operatorname{div} \mathbf{u}=0$ in (5) results in the following necessary condition for $q_{i}$ :

$$
(\mathbf{g}, \mathbf{n})_{\Gamma}=q_{1}+q_{2}+\ldots+q_{N}=0 .
$$

At the same time Theorem 1 is proved under more strict condition $\mathbf{g} \in \tilde{\mathbf{H}}^{1 / 2}(\Gamma)$ on $\mathbf{g}$ equivalent to $N$ conditions $q_{1}=0, q_{2}=0, \ldots, q_{N}=0$ for the vector $\mathbf{g}$. The latter is connected with the fact that the proof of Theorem 1 is based on the generalization of the Hopf's lemma see (Hopf, 1941). According to this generalized Hopf's lemma for any vector $\mathbf{g} \in \tilde{\mathbf{H}}^{1 / 2}(\Gamma)$ and any $\varepsilon>0$ there exists such a solenoidal expansion $\mathbf{u}_{0} \in \mathbf{H}_{\text {div }}^{1}(\Omega)$ into $\Omega$ for which

$$
\left|\left((\mathbf{v} \cdot \nabla) \mathbf{u}_{0}, \mathbf{v}\right)\right| \leq \varepsilon\|\mathbf{g}\|_{1 / 2, \Gamma}\|\mathbf{v}\|_{1}^{2} \forall \mathbf{v} \in \mathbf{V} .
$$

Using this lemma one can look for a weak solution of problem (5) at $\beta_{C}=0$ in the form $\mathbf{u}=\mathbf{u}_{0}+\tilde{\mathbf{u}}$, where $\tilde{\mathbf{u}} \in \mathbf{V}$ is a new unknown function and to obtain a "coercitive" nonlinear operator equation for function $\tilde{\mathbf{u}}$. The existence of the solution $\tilde{\mathbf{u}}$ of the latter equation can be proved using Schauder theorem (see e.g. (Alekseev \& Tereshko, 2008)). It should be noted that the question of Hopf's lemma validity and proof of the existence theorem for problem (5) at $\beta_{C}=0$ under fulfillment only condition $(\mathbf{g}, \mathbf{n})_{\Gamma}=0$ to the vector $\mathbf{g} \in \mathbf{H}^{1 / 2}(\Gamma)$ is till open. One can read about this problem (so called Leray problem) in more details in (Alekseev \& Tereshko, 2008, Appendix 5) and in (Pukhnachev, 2009; 2010).

The sufficient conditions of solvability of the stationary boundary value problem for the Navier-Stokes equations without the assumption $q_{i}=0, i=1, \ldots, N$ are stated in mentioned papers. Besides a detailed bibliography on Leray problem is provided. As to the general boundary value problem (1)-(3) for stationary heat and mass transfer equations, its uniqueness, even when the equalities $q_{i}=0$ hold, can be proved only in the case when values of thermal and diffusion Rayleigh numbers are small. Moreover the branching of stationary solutions is possible in the case of large values of Rayleigh numbers (Gershuni \& Zhukhovitskii, 1976; Joseph, 1976).

\section{General property of the optimality system solutions}

Let us consider control problem (21). Denote by $\left(\mathbf{x}_{1}, u_{1}\right) \equiv\left(\mathbf{u}_{1}, p_{1}, C_{1}, \mathbf{g}_{1}, \chi_{1}\right) \in X \times K$ its solution. By $\left(\mathbf{x}_{2}, u_{2}\right) \equiv\left(\mathbf{u}_{2}, p_{2}, C_{2}, \mathbf{g}_{2}, \chi_{2}\right) \in X \times K$ we denote a solution of problem

$$
\tilde{J}(\mathbf{x}, u)=\frac{\mu_{0}}{2} \tilde{I}(\mathbf{x})+\frac{\mu_{1}}{2}\|\mathbf{g}\|_{1 / 2, \Gamma}^{2}+\frac{\mu_{2}}{2}\|\chi\|_{\Gamma_{N}}^{2} \rightarrow \inf , F(\mathbf{x}, u)=0,(\mathbf{x}, u) \in X \times K,
$$

which is obtained from (21) by replacing a functional $I$ in (21) with another one $\tilde{I}$. In view of Theorem 1 the following estimates for pairs $\left(\mathbf{u}_{i}, p_{i}\right)$ hold

$$
\left\|\mathbf{u}_{i}\right\|_{1} \leq M_{\mathbf{u}}^{0}=\sup _{u \in K} M_{\mathbf{u}}\left(u_{0}, u\right),\left\|p_{i}\right\| \leq M_{p}^{0}=\sup _{u \in K} M_{p}\left(u_{0}, u\right),\left\|C_{i}\right\|_{1} \leq M_{C}^{0}=\sup _{u \in K} M_{C}\left(u_{0}, u\right) .
$$

We introduce "model" Reynolds number $\mathcal{R} e$, Rayleigh number $\mathcal{R} a$ and Prandtl number $\mathcal{P}$ by

$$
\mathcal{R} e=\frac{\gamma_{0} M_{\mathbf{u}}^{0}}{\delta_{0} v}, \mathcal{R} a=\frac{\gamma_{1}}{\delta_{0} v} \frac{\beta_{1} M_{C}^{0}}{\delta_{1} \lambda}, \mathcal{P}=\frac{\delta_{0} v}{\delta_{1} \lambda}
$$

and assume that

$$
\mathcal{R} e+\mathcal{R} a \equiv \frac{\gamma_{0} M_{\mathbf{u}}^{0}}{\delta_{0} v}+\frac{\gamma_{1}}{\delta_{0} v} \frac{\beta_{1} M_{C}^{0}}{\delta_{1} \lambda}<1 / 2
$$


Denote by $\left(1, \mathbf{y}_{i}^{*}\right) \equiv\left(1, \xi_{i}, \sigma_{i}, \zeta_{i}, \theta_{i}, \zeta_{i}^{c}\right), i=1,2$, the Lagrange multipliers corresponding to the solutions $\left(\mathbf{x}_{i}, u_{i}\right)$ (these multipliers are uniquely determined under condition (31)). By definition elements $\left(\xi_{i}, \sigma_{i}, \zeta_{i}, \theta_{i}, \zeta_{i}^{c}\right)$ satisfy relations

$$
\begin{gathered}
v a_{0}\left(\mathbf{w}, \xi_{i}\right)+c\left(\mathbf{u}_{i}, \mathbf{w}, \xi_{i}\right)+c\left(\mathbf{w}, \mathbf{u}_{i}, \xi_{i}\right)+\varkappa c_{1}\left(\mathbf{w}, C_{i}, \theta_{i}\right)+b\left(\mathbf{w}, \sigma_{i}\right)+\left\langle\zeta_{i}, \mathbf{w}\right\rangle_{\Gamma}= \\
=-\left(\mu_{0} / 2\right)\left\langle\left(I^{i}\right)_{\mathbf{u}}^{\prime}\left(\mathbf{x}_{i}\right), \mathbf{w}\right\rangle \forall \mathbf{w} \in \tilde{\mathbf{H}}^{1}(\Omega), b\left(\xi_{i}, r\right)=-\left(\mu_{0} / 2\right)\left(\left(I^{i}\right)_{p}^{\prime}\left(\mathbf{x}_{i}\right), r\right) \forall r \in L_{0}^{2}(\Omega), \\
\varkappa\left[\lambda a_{1}\left(\tau, \theta_{i}\right)+\left(k \tau, \theta_{i}\right)+c_{1}\left(\mathbf{u}_{i}, \tau, \theta_{i}\right)+\left\langle\zeta_{i}, \tau\right\rangle_{\Gamma_{D}}\right]-b_{1}\left(\tau, \xi_{i}\right)= \\
-\left(\mu_{0} / 2\right)\left\langle\left(I^{i}\right)_{C}^{\prime}\left(\mathbf{x}_{i}\right), \tau\right\rangle \forall \tau \in H^{1}(\Omega) .
\end{gathered}
$$

Here we renamed $I=I^{1}, \tilde{I}=I^{2}$. Let $\mathbf{g}=\mathbf{g}_{1}-\mathbf{g}_{2}, \chi=\chi_{1}-\chi_{2}$,

$\mathbf{u}=\mathbf{u}_{1}-\mathbf{u}_{2}, p=p_{1}-p_{2}, C=C_{1}-C_{2}, \xi=\xi_{1}-\xi_{2}, \sigma=\sigma_{1}-\sigma_{2}, \zeta=\zeta_{1}-\zeta_{2}, \theta=\theta_{1}-\theta_{2}, \zeta^{c}=\zeta_{2}^{c}-\zeta_{1}^{c}$.

Subtracting equations (17)-(19), written for $\mathbf{u}_{2}, p_{2}, C_{2}, u_{2}$, from corresponding equations (17)-(19) for $\mathbf{u}_{1}, p_{1}, C_{1}, u_{1}$ gives

$$
\begin{gathered}
v a_{0}(\mathbf{u}, \mathbf{v})+c\left(\mathbf{u}, \mathbf{u}_{1}, \mathbf{v}\right)+c\left(\mathbf{u}_{2}, \mathbf{u}, \mathbf{v}\right)+b(\mathbf{v}, p)-b_{1}(C, \mathbf{v})=0 \forall \mathbf{v} \in \mathbf{H}_{0}^{1}(\Omega), \\
\lambda a_{1}(C, S)+(k C, S)+c_{1}\left(\mathbf{u}, C_{1}, S\right)+c_{1}\left(\mathbf{u}_{2}, C, S\right)=(\chi, S)_{\Gamma_{N}} \forall S \in \mathcal{T}, \\
\operatorname{div} \mathbf{u}=0 \text { in } \Omega,\left.\mathbf{u}\right|_{\Gamma}=\mathbf{g},\left.C\right|_{\Gamma_{D}}=0 .
\end{gathered}
$$

Setting $\mathbf{g}=\mathbf{g}_{1}, \chi=\chi_{1}$ in (27) under $\lambda_{0}=1$, written for $\hat{\mathbf{g}}=\mathbf{g}_{2}, \hat{\chi}=\chi_{2}, \zeta=\zeta_{2}$ and setting $\mathbf{g}=\mathbf{g}_{2}, \chi=\chi_{2}$ in (27) written for $\hat{\mathbf{g}}=\mathbf{g}_{1}, \hat{\chi}=\chi_{1}, \zeta=\zeta_{1}$, we obtain $\mu_{1}\left(\mathbf{g}_{2}, \mathbf{g}\right)_{1 / 2, \Gamma}$ $\left\langle\zeta_{2}, \mathbf{g}\right\rangle_{\Gamma}+\mu_{2}\left(\chi_{2}, \chi\right)_{\Gamma_{N}}-\varkappa\left(\theta_{2}, \chi\right)_{\Gamma_{N}} \geq 0,-\mu_{1}\left(\mathbf{g}_{1}, \mathbf{g}\right)_{1 / 2, \Gamma}+\left\langle\zeta_{1}, \mathbf{g}\right\rangle_{\Gamma}-\mu_{2}\left(\chi_{1}, \chi\right)_{\Gamma_{N}}+\varkappa\left(\theta_{1}, \chi\right)_{\Gamma_{N}} \geq$ 0 . Adding up these inequalities yields the relation

$$
-\langle\zeta, \mathbf{g}\rangle_{\Gamma}-\varkappa(\theta, \chi)_{\Gamma_{N}} \leq-\mu_{1}\|\mathbf{g}\|_{1 / 2, \Gamma}^{2}-\mu_{2}\|\chi\|_{\Gamma_{N}}^{2}
$$

Subtract equations (32), (33) written for $\left(\mathbf{x}_{2}, u_{2}, \mathbf{y}_{2}^{*}\right)$ from corresponding equations for $\left(\mathbf{x}_{1}, u_{1}, \mathbf{y}_{1}^{*}\right)$. We obtain

$$
\begin{gathered}
v a_{0}(\mathbf{w}, \xi)+c\left(\mathbf{u}_{1}, \mathbf{w}, \xi\right)+c\left(\mathbf{u}, \mathbf{w}, \xi_{2}\right)+c\left(\mathbf{w}, \mathbf{u}_{1}, \xi\right)+c\left(\mathbf{w}, \mathbf{u}, \xi_{2}\right)+\varkappa c_{1}\left(\mathbf{w}, C_{1}, \theta\right)+\varkappa c_{1}\left(\mathbf{w}, C, \theta_{2}\right)+ \\
b(\mathbf{w}, \sigma)=-\langle\zeta, \mathbf{w}\rangle_{\Gamma}-\left(\mu_{0} / 2\right)\left\langle I_{\mathbf{u}}^{\prime}\left(\mathbf{x}_{1}\right)-\tilde{I}_{\mathbf{u}}^{\prime}\left(\mathbf{x}_{2}\right), \mathbf{w}\right\rangle \forall \mathbf{w} \in \tilde{\mathbf{H}}^{1}(\Omega) \\
\varkappa\left[\lambda a_{1}(\tau, \theta)+(k \tau, \theta)+c_{1}\left(\mathbf{u}_{1}, \tau, \theta\right)+c_{1}\left(\mathbf{u}, \tau, \theta_{2}\right)+\langle\zeta, \tau\rangle_{\Gamma_{D}}\right]-b_{1}(\tau, \xi)= \\
-\left(\mu_{0} / 2\right)\left\langle I_{C}^{\prime}\left(\mathbf{x}_{1}\right)-\tilde{I}_{C}^{\prime}\left(\mathbf{x}_{2}\right), \tau\right\rangle \forall \tau \in H^{1}(\Omega), \\
b(\xi, r)=-\left(\mu_{0} / 2\right)\left(I_{p}^{\prime}\left(\mathbf{x}_{1}\right)-\tilde{I}_{p}^{\prime}\left(\mathbf{x}_{2}\right), r\right) \forall r \in L_{0}^{2}(\Omega) .
\end{gathered}
$$


Set $\mathbf{w}=\mathbf{u}, \tau=C, r=p$ in (39), (40), (41) and add up the results. Taking into account (37) we obtain

$$
\begin{gathered}
v a_{0}(\mathbf{u}, \xi)+c\left(\mathbf{u}_{1}, \mathbf{u}, \xi\right)+2 c\left(\mathbf{u}, \mathbf{u}, \xi_{2}\right)+c\left(\mathbf{u}, \mathbf{u}_{1}, \xi\right)+\varkappa c_{1}\left(\mathbf{u}, C_{1}, \theta\right)+\varkappa c_{1}\left(\mathbf{u}, C, \theta_{2}\right)+\langle\zeta, \mathbf{g}\rangle_{\Gamma}+ \\
\varkappa\left[\lambda a_{1}(C, \theta)+(k C, \theta)+c_{1}\left(\mathbf{u}_{1}, C, \theta\right)+c_{1}\left(\mathbf{u}, C, \theta_{2}\right)\right]- \\
b_{1}(C, \xi)+b(\xi, p)=-\left(\mu_{0} / 2\right)\left\langle I_{\mathbf{u}}^{\prime}\left(\mathbf{x}_{1}\right)-\tilde{I}_{\mathbf{u}}^{\prime}\left(\mathbf{x}_{2}\right), \mathbf{u}\right\rangle-\left(\mu_{0} / 2\right)\left\langle I_{C}^{\prime}\left(\mathbf{x}_{1}\right)--\tilde{I}_{C}^{\prime}\left(\mathbf{x}_{2}\right), C\right\rangle- \\
-\left(\mu_{0} / 2\right)\left(I_{p}^{\prime}\left(\mathbf{x}_{1}\right)-\tilde{I}_{p}^{\prime}\left(\mathbf{x}_{2}\right), p\right) .
\end{gathered}
$$

Set $\mathbf{v}=\xi$ in (35), $S=\varkappa \theta$ in (36) and subtract the results from (42). Using (38) and identities

$$
\begin{gathered}
2 c\left(\mathbf{u}, \mathbf{u}, \xi_{2}\right)+c\left(\mathbf{u}_{1}, \mathbf{u}, \xi\right)-c\left(\mathbf{u}_{2}, \mathbf{u}, \xi\right)=2 c\left(\mathbf{u}, \mathbf{u}, \xi_{2}\right)+c(\mathbf{u}, \mathbf{u}, \xi)=c\left(\mathbf{u}, \mathbf{u}, \xi_{1}+\xi_{2}\right), \\
2 c_{1}\left(\mathbf{u}, C, \theta_{2}\right)+c_{1}\left(\mathbf{u}, C_{1}, \theta\right)-c_{1}\left(\mathbf{u}, C_{2}, \theta\right)=c_{1}\left(\mathbf{u}, C, \theta_{1}+\theta_{2}\right),
\end{gathered}
$$

we obtain

$$
\begin{gathered}
c\left(\mathbf{u}, \mathbf{u}, \xi_{1}+\xi_{2}\right)+\varkappa c_{1}\left(\mathbf{u}, C, \theta_{1}+\theta_{2}\right)+\left(\mu_{0} / 2\right)\left\langle I_{\mathbf{u}}^{\prime}\left(\mathbf{x}_{1}\right)-\tilde{I}_{\mathbf{u}}^{\prime}\left(\mathbf{x}_{2}\right), \mathbf{u}\right\rangle+ \\
\left(\mu_{0} / 2\right)\left\langle I_{C}^{\prime}\left(\mathbf{x}_{1}\right)-\tilde{I}_{C}^{\prime}\left(\mathbf{x}_{2}\right), C\right\rangle+\left(\mu_{0} / 2\right)\left(I_{p}^{\prime}\left(\mathbf{x}_{1}\right)-\tilde{I}_{p}^{\prime}\left(\mathbf{x}_{2}\right), p\right) \leq-\mu_{1}\|\mathbf{g}\|_{1 / 2, \Gamma}^{2}-\mu_{2}\|\chi\|_{\Gamma_{N}}^{2} .
\end{gathered}
$$

Thus the following result holds.

Theorem 6. Let under conditions of Theorem 4 for $I_{1}=I$ and $I_{2}=\tilde{I}$ pairs $\left(\mathbf{x}_{1}, u_{1}\right)=$ $\left(\mathbf{u}_{1}, p_{1}, C_{1}, \mathbf{g}_{1}, \chi_{1}\right) \in X \times K$ and $\left(\mathbf{x}_{2}, u_{2}\right)=\left(\mathbf{u}_{2}, p_{2}, C_{2}, \mathbf{g}_{2}, \chi_{2}\right) \in X \times K$ are solutions to problems (21) and (28) respectively, $\mathbf{y}_{i}^{*}=\left(\xi_{i}, \sigma_{i}, \zeta_{i}, \theta_{i}, \zeta_{i}^{c}\right), i=1,2$, are the Lagrange multipliers corresponding to these solutions $\left(\mathbf{x}_{i}, u_{i}\right)$. Then the relation (43) for differences $\mathbf{u}=\mathbf{u}_{1}-\mathbf{u}_{2}, p=p_{1}-p_{2}, C=C_{1}-C_{2}$, $\mathbf{g}=\mathbf{g}_{1}-\mathbf{g}_{2}, \chi=\chi_{1}-\chi_{2}$ holds.

Below we shall need some estimates of differences $\mathbf{u}=\mathbf{u}_{1}-\mathbf{u}_{2}$ and $p=p_{1}-p_{2}$ via differences $C=C_{1}-C_{2}$ and $\mathbf{g}=\mathbf{g}_{1}-\mathbf{g}_{2}$. In order to deduce them denote by $\mathbf{u}_{0} \in \mathbf{H}^{1}(\Omega)$ a function such that $\operatorname{div} \mathbf{u}_{0}=0,\left.\mathbf{u}_{0}\right|_{\Gamma}=\mathbf{g},\left\|\mathbf{u}_{0}\right\|_{1} \leq c_{0}\|\mathbf{g}\|_{1 / 2, \Gamma}$. Here a constant $c_{0}$ depends on $\Omega$. The existence of $\mathbf{u}_{0}$ follows from (Girault \& Raviart, 1986, p. 24). Set $\mathbf{u} \equiv \mathbf{u}_{1}-\mathbf{u}_{2}=\mathbf{u}_{0}+\tilde{\mathbf{u}}$ where $\tilde{\mathbf{u}} \in \mathbf{V}$ is a certain function. Set $\mathbf{u}=\mathbf{u}_{0}+\tilde{\mathbf{u}}, \mathbf{v}=\tilde{\mathbf{u}}$ in (35). Taking into account (8) we obtain

$$
v a_{0}(\tilde{\mathbf{u}}, \tilde{\mathbf{u}})=-v a_{0}\left(\mathbf{u}_{0}, \tilde{\mathbf{u}}\right)-c\left(\mathbf{u}_{0}, \mathbf{u}_{1}, \tilde{\mathbf{u}}\right)-c\left(\tilde{\mathbf{u}}, \mathbf{u}_{1}, \tilde{\mathbf{u}}\right)-c\left(\mathbf{u}_{2}, \mathbf{u}_{0}, \tilde{\mathbf{u}}\right)+b_{1}(C, \tilde{\mathbf{u}}) .
$$

Using estimates (10), (12), (14), (29) and this relation we deduce, that

$$
\delta_{0} v\|\tilde{\mathbf{u}}\|_{1}^{2} \leq v\left\|\mathbf{u}_{0}\right\|_{1}\|\tilde{\mathbf{u}}\|_{1}+\gamma_{0} M_{\mathbf{u}}^{0}\|\tilde{\mathbf{u}}\|_{1}^{2}+2 \gamma_{0} M_{\mathbf{u}}^{0}\left\|\mathbf{u}_{0}\right\|_{1}\|\tilde{\mathbf{u}}\|_{1}+\beta_{1}\|C\|_{1}\|\tilde{\mathbf{u}}\|_{1} .
$$

It follows from (31) that

$$
\left(\delta_{0} v / 2\right)<\delta_{0} v-\gamma_{0} M_{\mathbf{u}}^{0}-\frac{\beta_{1} \gamma_{1}}{\delta_{1} \lambda} M_{C}^{0} \leq \delta_{0} v-\gamma_{0} M_{\mathbf{u}}^{0}
$$

Rewriting (44) in view of (45) as

$$
\left(\delta_{0} v / 2\right)\|\tilde{\mathbf{u}}\|_{1}^{2} \leq\left(\delta_{0} v-\gamma_{0} M_{\mathbf{u}}^{0}\right)\|\tilde{\mathbf{u}}\|_{1}^{2} \leq\left(v+2 \gamma_{0} M_{\mathbf{u}}^{0}\right)\left\|\mathbf{u}_{0}\right\|_{1}\|\tilde{\mathbf{u}}\|_{1}+\beta_{1}\|C\|_{1}\|\tilde{\mathbf{u}}\|_{1},
$$


we obtain that

$$
\begin{aligned}
\|\tilde{\mathbf{u}}\|_{1} \leq & \left(2 / \delta_{0} v\right)\left(v+2 \gamma_{0} M_{\mathbf{u}}^{0}\right)\left\|\mathbf{u}_{0}\right\|_{1}+\left(2 \beta_{1} / \delta_{0} v\right)\|C\|_{1} \leq\left(2 \delta_{0}^{-1}+4 \mathcal{R} e\right)\left\|\mathbf{u}_{0}\right\|_{1}+ \\
& +\left(2 \beta_{1} / \delta_{0} v\right)\|C\|_{1} \leq 2 \mathcal{R}\left\|\mathbf{u}_{0}\right\|_{1}+\left(2 \beta_{1} / \delta_{0} v\right)\|C\|_{1}, \mathcal{R} \equiv \delta_{0}^{-1}+2 \mathcal{R} e .
\end{aligned}
$$

Taking into account that $\mathbf{u}=\mathbf{u}_{0}+\tilde{\mathbf{u}}$, we deduce the following estimate:

$$
\|\mathbf{u}\|_{1} \leq\left\|\mathbf{u}_{0}\right\|_{1}+\|\tilde{\mathbf{u}}\|_{1} \leq c_{0}(2 \mathcal{R}+1)\|\mathbf{g}\|_{1 / 2, \Gamma}+\left(2 \beta_{1} / \delta_{0} v\right)\|C\|_{1} .
$$

Analogous estimate holds and for pressure difference $p=p_{1}-p_{2}$. We make use inf-sup condition (16) for obtaining this estimate. By (16) for the function $p=p_{1}-p_{2}$ and any (small) number $\delta>0$ there exists a function $\mathbf{v}_{0} \in \mathbf{H}_{0}^{1}(\Omega), \mathbf{v}_{0} \neq 0$ such that $b\left(\mathbf{v}_{0}, p\right) \geq \beta_{0}\left\|\mathbf{v}_{0}\right\|_{1}\|p\|_{\text {, }}$ $\beta_{0}=(\beta-\delta)>0$. Set $\mathbf{v}=\mathbf{v}_{0}$ in (35). Using this estimate and (10), (12), (14) we have

$$
\beta_{0}\left\|\mathbf{v}_{0}\right\|_{1}\|p\| \leq b\left(\mathbf{v}_{0}, p\right) \leq\left(v+2 \gamma_{0} M_{\mathbf{u}}^{0}\right)\left\|\mathbf{v}_{0}\right\|_{1}\|\mathbf{u}\|_{1}+\beta_{1}\|C\|_{1}\left\|\mathbf{v}_{0}\right\|_{1} .
$$

As $\left\|\mathbf{v}_{0}\right\|_{1} \neq 0$ we deduce from this relation that

$$
\|p\| \leq \frac{v+2 \gamma_{0} M_{\mathbf{u}}^{0}}{\beta_{0}}\|\mathbf{u}\|_{1}+\frac{\beta_{1}}{\beta_{0}}\|C\|_{1}=\frac{\delta_{0} v}{\beta_{0}} \mathcal{R}\|\mathbf{u}\|_{1}+\frac{\beta_{1}}{\beta_{0}}\|C\|_{1} .
$$

Using (46) we obtain the following estimate for $\|p\|$ :

$$
\|p\| \leq \frac{\delta_{0} v}{\beta_{0}} c_{0} \mathcal{R}(2 \mathcal{R}+1)\|\mathbf{g}\|_{1 / 2, \Gamma}+\frac{\beta_{1}}{\beta_{0}}(2 \mathcal{R}+1)\|C\|_{1} .
$$

Based on Theorem 6 and estimates (46)-(48) we establish in the next section sufficient conditions to input data which provide uniqueness and stability of the solution $(\hat{\mathbf{x}}, \hat{u})$ to problem (21) for a number of concrete cost functionals and controls.

Remark 3. Let us note that if $u=\mathbf{g}$ (or $u=\chi$ ) then this boundary control problem can be considered as a particular case of the general boundary control problem (21) corresponding to situation when $K_{2}\left(\right.$ or $\left.K_{1}\right)$ is singleton: $K_{2}=\{\chi\}$ (or $K_{1}=\{\mathbf{g}\}$ ).

\section{Uniqueness and stability of solutions of boundary control problems}

In this section we firstly consider the problem (21) in the case where $I=I_{1}$ and $u=\mathbf{g} \in K_{1}$, i.e. we consider one-parameter boundary control problem

$$
J(\mathbf{x}, \mathbf{g}) \equiv \frac{\mu_{0}}{2}\left\|\mathbf{v}-\mathbf{v}_{d}\right\|_{Q}^{2}+\frac{\mu_{1}}{2}\|\mathbf{g}\|_{1 / 2, \Gamma}^{2} \rightarrow \inf , F(\mathbf{x}, \mathbf{g})=0, \mathbf{x}=(\mathbf{v}, q, S) \in X, \mathbf{g} \in K_{1} .
$$

Let $\left(\mathbf{x}_{1}, u_{1}\right) \equiv\left(\mathbf{u}_{1}, p_{1}, C_{1}, \mathbf{g}_{1}\right)$ be a solution to problem (49) which corresponds to a function $\mathbf{v}_{d} \equiv \mathbf{u}_{d}^{(1)} \in \mathbf{L}^{2}(Q),\left(\mathbf{x}_{2}, u_{2}\right) \equiv\left(\mathbf{u}_{2}, p_{2}, C_{2}, \mathbf{g}_{2}\right)$ be a solution to problem (49) which corresponds to another function $\tilde{\mathbf{v}}_{d} \equiv \mathbf{u}_{d}^{(2)} \in \mathbf{L}^{2}(Q)$. Setting $\mathbf{g}=\mathbf{g}_{1}-\mathbf{g}_{2}, \mathbf{u}_{d}=\mathbf{u}_{d}^{(1)}-\mathbf{u}_{d}^{(2)}$ in addition to (34), we note that

$$
\begin{gathered}
\left\langle\left(I_{1}\right)_{\mathbf{u}}^{\prime}\left(\mathbf{x}_{i}\right), \mathbf{w}\right\rangle=2\left(\mathbf{u}_{i}-\mathbf{u}_{d}, \mathbf{w}\right)_{Q},\left\langle\left(I_{1}\right)_{\mathbf{u}}^{\prime}\left(\mathbf{x}_{1}\right)-\left(I_{1}\right)_{\mathbf{u}}^{\prime}\left(\mathbf{x}_{2}\right), \mathbf{u}\right\rangle=2\left(\mathbf{u}-\mathbf{u}_{d}, \mathbf{u}\right)_{Q}= \\
2\left(\|\mathbf{u}\|_{Q}^{2}-\left(\mathbf{u}, \mathbf{u}_{d}\right)_{Q}\right),\left(I_{1}\right)_{C}^{\prime}=0 .
\end{gathered}
$$


The relations (35), (37) for problem (49) do not change while identities (36), (32), (33) and the main inequality (43) take by (50) and remark 1 a form

$$
\lambda a_{1}(C, S)+(k C, S)+c_{1}\left(\mathbf{u}, C_{1}, S\right)+c_{1}\left(\mathbf{u}_{2}, C, S\right)=0 \forall S \in \mathcal{T},
$$

$$
\begin{aligned}
& v a_{0}\left(\mathbf{w}, \xi_{i}\right)+ c\left(\mathbf{u}_{i}, \mathbf{w}, \xi_{i}\right)+c\left(\mathbf{w}, \mathbf{u}_{i}, \xi_{i}\right)+\varkappa c_{1}\left(\mathbf{w}, C_{i}, \theta_{i}\right)+b\left(\mathbf{w}, \sigma_{i}\right)+\left\langle\zeta_{i}, \mathbf{w}\right\rangle_{\Gamma}= \\
&-\mu_{0}\left(\mathbf{u}_{i}-\mathbf{u}_{d}^{(i)}, \mathbf{w}\right)_{Q} \forall \mathbf{w} \in \tilde{\mathbf{H}}^{1}(\Omega), \quad \xi_{i} \in \mathbf{V}, i=1,2, \\
& \varkappa\left[\lambda \tilde{a}\left(\tau, \theta_{i}\right)+\left(k \tau, \theta_{i}\right)+c_{1}\left(\mathbf{u}_{i}, \tau, \theta_{i}\right)+\left\langle\zeta_{i}, \tau\right\rangle_{\Gamma_{D}}\right]-b_{1}\left(\tau, \xi_{i}\right)=0 \forall \tau \in H^{1}(\Omega), \\
& c\left(\mathbf{u}, \mathbf{u}, \xi_{1}+\xi_{2}\right)+\varkappa c_{1}\left(\mathbf{u}, C, \theta_{1}+\theta_{2}\right)+\mu_{0}\left(\|\mathbf{u}\|_{Q}^{2}-\left(\mathbf{u}, \mathbf{u}_{d}\right)_{Q}\right) \leq-\mu_{1}\|\mathbf{g}\|_{1 / 2, \Gamma}^{2} .
\end{aligned}
$$

It follows from (37) that $C \in \mathcal{T}$. Set $S=C$ in (51). Using (9) we obtain that

$$
\lambda a_{1}(C, C)+(k C, C)=-c_{1}\left(\mathbf{u}, C_{1}, C\right) .
$$

It follows from (13), (14), (29) that

$$
\left|c_{1}\left(\mathbf{u}, C_{1}, C\right)\right| \leq \gamma_{1} M_{C}^{0}\|\mathbf{u}\|_{1}\|C\|_{1},\left|b_{1}(C, \mathbf{u})\right| \leq \beta_{1}\|\mathbf{u}\|_{1}\|C\|_{1} .
$$

Taking into account (11), (56), we obtain from (55) that $\delta_{1} \lambda\|C\|^{2} \leq \gamma_{1} M_{C}^{0}\|\mathbf{u}\|_{1}\|C\|_{1}$. From this inequality we deduce the following estimate for $\|C\|_{1}$ :

$$
\|C\|_{1} \leq \frac{\gamma_{1} M_{C}^{0}}{\delta_{1} \lambda}\|\mathbf{u}\|_{1}
$$

Using (46), (57) and (30) we have

$$
\|\mathbf{u}\|_{1} \leq c_{0}(2 \mathcal{R}+1)\|\mathbf{g}\|_{1 / 2, \Gamma}+\frac{2 \beta_{1}}{\delta_{0} v} \frac{\gamma_{1} M_{C}^{0}}{\delta_{1} \lambda}\|\mathbf{u}\|_{1}=c_{0}(2 \mathcal{R}+1)\|\mathbf{g}\|_{1 / 2, \Gamma}+2 \mathcal{R} a\|\mathbf{u}\|_{1} .
$$

It follows from (58) and (30) that $(1-2 \mathcal{R} a)\|\mathbf{u}\|_{1} \leq c_{0}(2 \mathcal{R}+1)\|\mathbf{g}\|_{1 / 2, \Gamma}$. Taking into account that $2 \mathcal{R} a<1$ by (31) we obtain from this estimate, (57) and (48) that

$$
\begin{gathered}
\|\mathbf{u}\|_{1} \leq \frac{c_{0}(2 \mathcal{R}+1)}{1-2 \mathcal{R} a}\|\mathbf{g}\|_{1 / 2, \Gamma} \\
\|C\|_{1} \leq \frac{c_{0}(2 \mathcal{R}+1)}{(1-2 \mathcal{R} a)} \frac{\gamma_{1} M_{C}^{0}}{\delta_{1} \lambda}\|\mathbf{g}\|_{1 / 2, \Gamma},\|p\| \leq \frac{\delta_{0} v c_{0}(2 \mathcal{R}+1)(\mathcal{R}+\mathcal{R} a)}{\beta_{0}(1-2 \mathcal{R} a)}\|\mathbf{g}\|_{1 / 2, \Gamma} .
\end{gathered}
$$

Set $\mathbf{w}=\xi_{i}, \tau=\theta_{i}$ in (52), (53). Using (8), (9) and conditions $\xi_{i} \in \mathbf{V}, \theta_{i} \in \mathcal{T}$ we deduce that

$$
\begin{gathered}
v a_{0}\left(\xi_{i}, \xi_{i}\right)=-c\left(\xi_{i}, \mathbf{u}_{i}, \xi_{i}\right)-\varkappa c_{1}\left(\xi_{i}, C_{i}, \theta_{i}\right)-\mu_{0}\left(\mathbf{u}_{i}-\mathbf{u}_{d}^{(i)}, \xi_{i}\right)_{Q}, \\
\varkappa\left[\lambda a_{1}\left(\theta_{i}, \theta_{i}\right)+\left(k \theta_{i}, \theta_{i}\right)\right]=b_{1}\left(\theta_{i}, \xi_{i}\right), i=1,2 .
\end{gathered}
$$

It follows from (10)-(14), (7), (29) that

$$
a_{0}\left(\xi_{i}, \xi_{i}\right) \geq \delta_{0}\left\|\xi_{i}\right\|_{1}^{2},\left|c\left(\xi_{i}, \mathbf{u}_{i}, \xi_{i}\right)\right| \leq \gamma_{0}\left\|\mathbf{u}_{i}\right\|_{1}\left\|\xi_{i}\right\|_{1}^{2} \leq \gamma_{0} M_{\mathbf{u}}^{0}\left\|\xi_{i}\right\|_{1}^{2},
$$




$$
\begin{gathered}
a_{1}\left(\theta_{i}, \theta_{i}\right) \geq \delta_{1}\left\|\theta_{i}\right\|_{1}^{2},\left|b_{1}\left(\theta_{i}, \xi_{i}\right)\right| \leq \beta_{1}\left\|\theta_{i}\right\|_{1}\left\|\xi_{i}\right\|_{1},\left|c_{1}\left(\xi_{i}, C_{i}, \theta_{i}\right)\right| \leq \gamma_{1} M_{C}^{0}\left\|\xi_{i}\right\|_{1}\left\|\theta_{i}\right\|_{1}, \\
\left|\left(\mathbf{u}_{i}-\mathbf{u}_{d}^{(i)}, \xi_{i}\right)_{Q}\right| \leq\left\|\mathbf{u}_{i}-\mathbf{u}_{d}^{(i)}\right\|_{Q}\left\|\xi_{i}\right\|_{Q} \leq c_{Q}\left(c_{Q} M_{\mathbf{u}}^{0}+\left\|\mathbf{u}_{d}^{(i)}\right\|_{Q}\right)\left\|\xi_{i}\right\|_{1} .
\end{gathered}
$$

Taking into account (63)-(65) we deduce from (61) and (62) that

$$
\left\|\theta_{i}\right\|_{1} \leq \frac{\beta_{1}}{\delta_{1} \lambda \varkappa}\left\|\xi_{i}\right\|_{1},\left(\delta_{0} v-\gamma_{0} M_{\mathbf{u}}^{0}-\frac{\beta_{1} \gamma_{1}}{\delta_{1} \lambda} M_{C}^{0}\right)\left\|\xi_{i}\right\|_{1}^{2} \leq \mu_{0} c_{Q}\left(c_{Q} M_{\mathbf{u}}^{0}+\left\|\mathbf{u}_{d}^{(i)}\right\|_{Q}\right)\left\|\xi_{i}\right\|_{1} .
$$

Combining these inequalities with (45) and (30) gives

$$
\left\|\xi_{i}\right\|_{1} \leq \frac{2 \mu_{0} \gamma_{3}}{\gamma_{0}}\left(\mathcal{R} e+\mathcal{R} e^{0}\right),\left\|\theta_{i}\right\|_{1} \leq \frac{\beta_{1}}{\delta_{1} \lambda \varkappa} \frac{2 \mu_{0} \gamma_{3}}{\gamma_{0}}\left(\mathcal{R} e+\mathcal{R} e^{0}\right),
$$

where

$$
\gamma_{3}=c_{Q}^{2}, \mathcal{R} e^{0}=\frac{\gamma_{0}}{\delta_{0} v c_{Q}} \max \left(\left\|\mathbf{u}_{d}^{(1)}\right\|_{Q},\left\|\mathbf{u}_{d}^{(2)}\right\|_{Q}\right) .
$$

Taking into account (12), (13), (57), (66) and (30) we have

$$
\begin{gathered}
\left|c\left(\mathbf{u}, \mathbf{u}, \xi_{1}+\xi_{2}\right)\right| \leq \gamma_{0}\|\mathbf{u}\|_{1}^{2}\left(\left\|\xi_{1}\right\|_{1}+\left\|\xi_{2}\right\|_{1}\right) \leq 4 \mu_{0} \gamma_{3}\left(\mathcal{R} e+\mathcal{R} e^{0}\right)\|\mathbf{u}\|_{1}^{2}, \\
\varkappa\left|c_{1}\left(\mathbf{u}, C, \theta_{1}+\theta_{2}\right)\right| \leq \varkappa \gamma_{1}\|\mathbf{u}\|_{1}\|C\|_{1}\left(\left\|\theta_{1}\right\|_{1}+\left\|\theta_{2}\right\|_{1}\right) \leq \\
\frac{\gamma_{1} M_{C}^{0}}{\delta_{1} \lambda} \frac{\gamma_{1} \beta_{1}}{\delta_{1} \lambda} \frac{4 \mu_{0} \gamma_{3}\left(\mathcal{R} e+\mathcal{R} e^{0}\right)}{\gamma_{0}}\|\mathbf{u}\|_{1}^{2}=4 \mu_{0} \gamma_{3}\left(\mathcal{R} e+\mathcal{R} e^{0}\right) \frac{\gamma_{1}}{\gamma_{0}} \mathcal{P} \mathcal{R} a\|\mathbf{u}\|_{1}^{2}
\end{gathered}
$$

which yields

$$
\left|c\left(\mathbf{u}, \mathbf{u}, \xi_{1}+\xi_{2}\right)+\varkappa c_{1}\left(\mathbf{u}, C, \theta_{1}+\theta_{2}\right)\right| \leq 4 \mu_{0} \gamma_{3}\left(\mathcal{R} e+\mathcal{R} e^{0}\right)\left[1+\left(\gamma_{1} / \gamma_{0}\right) \mathcal{P} \mathcal{R} a\right]\|\mathbf{u}\|_{1}^{2} .
$$

Using (59) we deduce from (68) that

$$
\begin{gathered}
\left|c\left(\mathbf{u}, \mathbf{u}, \xi_{1}+\xi_{2}\right)+\varkappa c_{1}\left(\mathbf{u}, C, \theta_{1}+\theta_{2}\right)\right| \leq \\
\leq \frac{4 \mu_{0} \gamma_{3} c_{0}^{2}(2 \mathcal{R}+1)^{2}\left(\mathcal{R} e+\mathcal{R} e^{0}\right)\left[1+\left(\gamma_{1} / \gamma_{0}\right) \mathcal{P} \mathcal{R} a\right]}{(1-2 \mathcal{R} a)^{2}}\|\mathbf{g}\|_{1 / 2, \Gamma}^{2} .
\end{gathered}
$$

Let input data for problem (49) and parameters $\mu_{0}, \mu_{1}$ be such that

$$
(1-\varepsilon) \mu_{1} \geq \frac{4 \mu_{0} \gamma_{3} c_{0}^{2}(2 \mathcal{R}+1)^{2}\left(\mathcal{R} e+\mathcal{R} e^{0}\right)\left[1+\left(\gamma_{1} / \gamma_{0}\right) \mathcal{P} \mathcal{R} a\right]}{(1-2 \mathcal{R} a)^{2}}, \varepsilon=\text { const }>0 .
$$

Here and further $\varepsilon>0$ is a (small) constant. In view of (70) we find from (69) that

$$
\left|c\left(\mathbf{u}, \mathbf{u}, \xi_{1}+\xi_{2}\right)+\varkappa c_{1}\left(\mathbf{u}, C, \theta_{1}+\theta_{2}\right)\right| \leq(1-\varepsilon) \mu_{1}\|\mathbf{g}\|_{1 / 2, \Gamma}^{2} .
$$

Taking into account (71) we come from (54) to the inequality

$$
\mu_{0}\left(\|\mathbf{u}\|_{Q}^{2}-\left(\mathbf{u}, \mathbf{u}_{d}\right)_{Q}\right) \leq-c\left(\mathbf{u}, \mathbf{u}, \xi_{1}+\xi_{2}\right)-\varkappa c_{1}\left(\mathbf{u}, C, \theta_{1}+\theta_{2}\right)-\mu_{1}\|\mathbf{g}\|_{1 / 2, \Gamma}^{2} \leq-\varepsilon \mu_{1}\|\mathbf{g}\|_{1 / 2, \Gamma}^{2} .
$$


It follows from (72) that $\|\mathbf{u}\|_{Q}^{2} \leq\left(\mathbf{u}, \mathbf{u}_{d}\right)_{Q} \leq\|\mathbf{u}\|_{Q}\left\|\mathbf{u}_{d}\right\|_{Q}$, which yields $\|\mathbf{u}\|_{Q} \leq\left\|\mathbf{u}_{d}\right\|_{Q}$. As $\mathbf{u}=\mathbf{u}_{1}-\mathbf{u}_{2}, \mathbf{u}_{d}=\mathbf{u}_{d}^{(1)}-\mathbf{u}_{d}^{(2)}$ we deduce the following estimate:

$$
\left\|\mathbf{u}_{1}-\mathbf{u}_{2}\right\|_{Q} \leq\left\|\mathbf{u}_{d}^{(1)}-\mathbf{u}_{d}^{(2)}\right\|_{Q}
$$

The estimate (73) in the case where $Q=\Omega$ has the sense of the stability estimate of the component $\hat{\mathbf{u}}$ of the solution $(\hat{\mathbf{u}}, \hat{p}, \hat{C}, \hat{\mathbf{g}})$ to problem (49) with respect to small disturbances in the $\mathbf{L}^{2}(\Omega)$-norm of the function $\mathbf{v}_{d} \in \mathbf{L}^{2}(\Omega)$ which enters into the expression for the functional $I_{1}$ in (22). In the case where $\mathbf{u}_{d}^{(1)}=\mathbf{u}_{d}^{(2)}$ it follows from (73) that $\mathbf{u}_{1}=\mathbf{u}_{2}$. This yields together with (57), (47) and condition $\left.\mathbf{u}\right|_{\Gamma}=\mathbf{g} \equiv \mathbf{g}_{1}-\mathbf{g}_{2}$ in (35) that $C_{1}=C_{2}, p_{1}=p_{2}$ and $\mathbf{g}_{1}=\mathbf{g}_{2}$. The latter means the uniqueness of the solution to problem (49) when $Q=\Omega$ and (70) holds.

We note that the uniqueness and stability of the solution to problem (49) under condition (70) take place and in the case where $Q \subset \Omega$, i.e. $Q$ is a part of $\Omega$. In order to prove this fact let us consider the inequality (72). Using (73) rewrite it in the form

$$
\begin{aligned}
\varepsilon \mu_{1}\|\mathbf{g}\|_{1 / 2, \Gamma}^{2} & \leq c\left(\mathbf{u}, \mathbf{u}, \xi_{1}+\xi_{2}\right)+\varkappa c_{1}\left(\mathbf{u}, C, \theta_{1}+\theta_{2}\right)+\mu_{1}\|\mathbf{g}\|_{1 / 2, \Gamma}^{2} \leq \\
& \leq-\mu_{0}\|\mathbf{u}\|_{Q}^{2}+\mu_{0}\|\mathbf{u}\|_{Q}\left\|\mathbf{u}_{d}\right\|_{Q} \leq \mu_{0}\left\|\mathbf{u}_{d}\right\|_{Q}^{2} .
\end{aligned}
$$

From this relation, (59) and (60) we deduce the following stability estimates:

$$
\begin{gathered}
\left\|\mathbf{g}_{1}-\mathbf{g}_{2}\right\|_{1 / 2, \Gamma} \leq \sqrt{\frac{\mu_{0}}{\varepsilon \mu_{1}}}\left\|\mathbf{u}_{d}^{(1)}-\mathbf{u}_{d}^{(2)}\right\|_{\mathbf{H}^{s}(Q)},\left\|\mathbf{u}_{1}-\mathbf{u}_{2}\right\|_{1} \leq \frac{c_{0}(2 \mathcal{R}+1)}{1-2 \mathcal{R} a} \sqrt{\frac{\mu_{0}}{\varepsilon \mu_{1}}}\left\|\mathbf{u}_{d}^{(1)}-\mathbf{u}_{d}^{(2)}\right\|_{\mathbf{H}^{s}(Q)}, \\
\left\|C_{1}-C_{2}\right\|_{1} \leq \frac{\gamma_{1} M_{C}^{0} c_{0}(2 \mathcal{R}+1)}{\delta_{1} \lambda(1-2 \mathcal{R} a)} \sqrt{\frac{\mu_{0}}{\varepsilon \mu_{1}}}\left\|\mathbf{u}_{d}^{(1)}-\mathbf{u}_{d}^{(2)}\right\|_{\mathbf{H}^{s}(Q)}, \\
\left\|p_{1}-p_{2}\right\| \leq \\
\frac{\delta_{0} v c_{0}(2 \mathcal{R}+1)(\mathcal{R}+\mathcal{R} a)}{\beta_{0}(1-2 \mathcal{R} a)} \sqrt{\frac{\mu_{0}}{\varepsilon \mu_{1}}}\left\|\mathbf{u}_{d}^{(1)}-\mathbf{u}_{d}^{(2)}\right\|_{\mathbf{H}^{s}(Q)}\left(\mathcal{R} \equiv \delta_{0}^{-1}+2 \mathcal{R} e\right)
\end{gathered}
$$

where $s=0$. Thus we have proved the following theorem.

Theorem 7. Let under conditions (i), (ii), (j) and (31) the quadruple $\left(\mathbf{u}_{i}, p_{i}, C_{i}, \mathbf{g}_{i}\right)$ be the solution to problem (49) corresponding to a given function $\mathbf{u}_{d}^{(i)} \in \mathbf{L}^{2}(Q), i=1,2$, and the condition (70) holds where $\gamma_{3}$ and $\mathcal{R} e^{0}$ are defined in (67). Then stability estimates (73) and (74) under $s=0$ hold true.

We emphasize that the uniqueness and stability of the solution to problem (49) both under $Q=\Omega$, and under $Q \subset \Omega$ is proved only if parameter $\mu_{1}$ in (49) is positive and satisfies (70). This means that term $\left(\mu_{1} / 2\right)\|\mathbf{g}\|_{1 / 2, \Gamma}^{2}$ in the expression for the minimized functional $J$ in (49) has a regularizing effect on control problem (49).

In the same manner one can study uniqueness and stability of solutions to boundary control problems for another cost functionals depending on the velocity $\mathbf{u}$. Let us consider for example the control problem

$$
J(\mathbf{x}, \mathbf{g}) \equiv \frac{\mu_{0}}{2}\left\|\mathbf{v}-\mathbf{v}_{d}\right\|_{1, Q}^{2}+\frac{\mu_{1}}{2}\|\mathbf{g}\|_{1 / 2, \Gamma}^{2} \rightarrow \inf , F(\mathbf{x}, \mathbf{g})=0, \mathbf{x}=(\mathbf{v}, q, S) \in X, \mathbf{g} \in K_{1},
$$

which corresponds to the cost functional $I_{2}(\mathbf{v})=\left\|\mathbf{v}-\mathbf{v}_{d}\right\|_{1, Q}^{2}$. Denoting by $\left(\mathbf{x}_{i}, u_{i}\right) \equiv$ $\left(\mathbf{u}_{i}, p_{i}, C_{i}, \mathbf{g}_{i}\right)$ the solution to problem (75) corresponding to a function $\mathbf{v}_{d} \equiv \mathbf{u}_{d}^{(i)} \in \mathbf{H}^{1}(Q)$, $i=1,2$, and setting $\mathbf{g}=\mathbf{g}_{1}-\mathbf{g}_{2}, \mathbf{u}_{d}=\mathbf{u}_{d}^{(1)}-\mathbf{u}_{d}^{(2)}$ in addition to (34) we note that

$$
\left\langle\left(I_{2}\right)_{\mathbf{u}}^{\prime}\left(\mathbf{x}_{i}\right), \mathbf{w}\right\rangle=2\left(\mathbf{u}_{i}-\mathbf{u}_{d}^{(i)}, \mathbf{w}\right)_{1, Q},\left\langle\left(I_{2}\right)_{\mathbf{u}}^{\prime}\left(\mathbf{x}_{1}\right)-\left(I_{2}\right)_{\mathbf{u}}^{\prime}\left(\mathbf{x}_{2}\right), \mathbf{u}\right\rangle=2\left(\mathbf{u}-\mathbf{u}_{d}, \mathbf{u}\right)_{1, Q}
$$




$$
=2\left(\|\mathbf{u}\|_{1, Q}^{2}-\left(\mathbf{u}, \mathbf{u}_{d}\right)_{1, Q}\right),\left(I_{2}\right)_{C}^{\prime}=0 .
$$

In view of (76) relations (35), (37), (51), (53), (62) and estimates (57), (59), (60) do not change while (43) and (32) under $\mathbf{w}=\xi_{i}$ take instead of (54), (61) the form

$$
\begin{gathered}
c\left(\mathbf{u}, \mathbf{u}, \xi_{1}+\xi_{2}\right)+\varkappa c_{1}\left(\mathbf{u}, C, \theta_{1}+\theta_{2}\right)+\mu_{0}\left(\|\mathbf{u}\|_{1, Q}-\left(\mathbf{u}, \mathbf{u}_{d}\right)_{1, Q}\right) \leq-\mu_{1}\|\mathbf{g}\|_{1 / 2, \Gamma}^{2}, \\
v a\left(\xi_{i}, \xi_{i}\right)=-c\left(\xi_{i}, \mathbf{u}_{i}, \xi_{i}\right)-\varkappa c_{1}\left(\xi_{i}, C_{i}, \theta_{i}\right)-\mu_{0}\left(\mathbf{u}_{i}-\mathbf{u}_{d}^{(i)}, \xi_{i}\right)_{1, Q}, \quad \xi_{i} \in \mathbf{V} .
\end{gathered}
$$

Using the estimates (29) we deduce (instead of (65)) that

$$
\left|\left(\mathbf{u}_{i}-\mathbf{u}_{d}^{(i)}, \xi_{i}\right)_{1, Q}\right| \leq\left\|\mathbf{u}_{i}-\mathbf{u}_{d}^{(i)}\right\|_{1, Q}\left\|\xi_{i}\right\|_{1, Q} \leq\left(M_{\mathbf{u}}^{0}+\left\|\mathbf{u}_{d}^{(i)}\right\|_{1, Q}\right)\left\|\xi_{i}\right\|_{1} .
$$

Proceeding as above we obtain estimates (66) for $\left\|\xi_{i}\right\|_{1},\left\|\theta_{i}\right\|_{1}$ and inequality (69) where

$$
\gamma_{3}=1, \mathcal{R} e^{0}=\left(\gamma_{0} / \delta_{0} v\right) \max \left(\left\|\mathbf{u}_{d}^{(1)}\right\|_{1, Q},\left\|\mathbf{u}_{d}^{(2)}\right\|_{1, Q}\right) .
$$

Let us assume that condition (70) takes place where $\gamma_{3}$ and $\mathcal{R} e^{0}$ are defined in (79). Using (70) we deduce (71). Taking into account (71) we obtain from (77) that

$\mu_{0}\left(\|\mathbf{u}\|_{1, Q}^{2}-\left(\mathbf{u}, \mathbf{u}_{d}\right)_{1, Q}\right) \leq-c\left(\mathbf{u}, \mathbf{u}, \xi_{1}+\xi_{2}\right)-\varkappa c_{1}\left(\mathbf{u}, C, \theta_{1}+\theta_{2}\right)-\mu_{1}\|\mathbf{g}\|_{1 / 2, \Gamma}^{2} \leq-\varepsilon \mu_{1}\|\mathbf{g}\|_{1 / 2, \Gamma}^{2}$.

It follows from (80) that $\|\mathbf{u}\|_{1, Q}^{2} \leq\left(\mathbf{u}, \mathbf{u}_{d}\right)_{1, Q}$ which yields $\|\mathbf{u}\|_{1, Q} \leq\left\|\mathbf{u}_{d}\right\|_{1, Q}$ or

$$
\left\|\mathbf{u}_{1}-\mathbf{u}_{2}\right\|_{1, Q} \leq\left\|\mathbf{u}_{d}^{(1)}-\mathbf{u}_{d}^{(2)}\right\|_{1, Q}
$$

In the case where $Q=\Omega$ we deduce from (81), relation $\left.\mathbf{u}\right|_{\Gamma}=\mathbf{g}=\mathbf{g}_{1}-\mathbf{g}_{2}$, (7), (57) and (47) the following estimates:

$$
\begin{gathered}
\left\|\mathbf{u}_{1}-\mathbf{u}_{2}\right\|_{1} \leq\left\|\mathbf{u}_{d}^{(1)}-\mathbf{u}_{d}^{(2)}\right\|_{1},\left\|\mathbf{g}_{1}-\mathbf{g}_{2}\right\|_{1 / 2, \Gamma} \leq c_{\Gamma}\left\|\mathbf{u}_{d}^{(1)}-\mathbf{u}_{d}^{(2)}\right\|_{1}, \\
\left\|C_{1}-C_{2}\right\|_{1} \leq \frac{\gamma_{1} M_{C}^{0}}{\delta_{1} \lambda}\left\|\mathbf{u}_{d}^{(1)}-\mathbf{u}_{d}^{(2)}\right\|_{1},\left\|p_{1}-p_{2}\right\| \leq \frac{\delta_{0} v(\mathcal{R}+\mathcal{R} a)}{\beta_{0}}\left\|\mathbf{u}_{d}^{(1)}-\mathbf{u}_{d}^{(2)}\right\|_{1} .
\end{gathered}
$$

The estimates (82) have the sense of stability estimates for the solution $(\hat{\mathbf{u}}, \hat{p}, \hat{C}, \hat{\mathbf{g}})$ to problem (75) under $Q=\Omega$ with respect to small disturbances in $\mathbf{H}^{1}(\Omega)$-norm of the function $\mathbf{v}_{d}$ which enters into the expression for the functional $I_{2}$. In the case where $\mathbf{u}_{d}^{(1)}=\mathbf{u}_{d}^{(2)}$ we deduce from (82) that $\mathbf{u}_{1}=\mathbf{u}_{2}, \mathbf{g}_{1}=\mathbf{g}_{2}, C_{1}=C_{2}, p_{1}=p_{2}$ which means the uniqueness of solution to control problem (75) under $Q=\Omega$. If $Q \subset \Omega$ the estimates (82) do not hold true but using (80) one can obtain more rough estimates of kind (74) instead of them. In fact rewriting (80) in view of (81) in the form

$$
\varepsilon \mu_{1}\|\mathbf{g}\|_{1 / 2, \Gamma}^{2} \leq-\mu_{0}\|\mathbf{u}\|_{1, Q}^{2}+\mu_{0}\|\mathbf{u}\|_{1, Q}\left\|\mathbf{u}_{d}\right\|_{1, Q} \leq \mu_{0}\left\|\mathbf{u}_{d}\right\|_{1, Q}^{2}
$$

and using (59), (60) we come to to the estimates (74) under $s=1$. Thus we have proved the following result.

Theorem 8. Let under conditions (i), (ii), (j) and (31) the quadruple $\left(\mathbf{u}_{i}, p_{i}, C_{i}, \mathbf{g}_{i}\right)$ be a solution to problem (75) corresponding to a given function $\mathbf{u}_{d}^{(i)} \in \mathbf{H}^{1}(Q), i=1,2$, and the condition (70) holds 
where $\gamma_{3}$ and $\mathcal{R} e^{0}$ are defined in (79). Then stability estimates (81) and (74) under $s=1$ hold true. Furthermore estimates (82) hold if $Q=\Omega$.

We again note that the uniqueness and stability of the solution to problem (75) both under $Q=\Omega$ and under $Q \subset \Omega$ is proved above under condition that the parameter $\mu_{1}$ in (75) satisfies (70). We can not prove the stability of the solution to problem (75) as well as to problem (49) in the case where $\mu_{1}=0$. But we can establish the local uniqueness of the solution to problem (75) under $\mu_{1}=0$ in the case where $Q=\Omega$. In fact setting $\mu_{1}=0, Q=\Omega, \mathbf{u}_{d}^{(1)}=\mathbf{u}_{d}^{(2)}$ in (77) we obtain the inequality

$$
c\left(\mathbf{u}, \mathbf{u}, \xi_{1}+\xi_{2}\right)+\varkappa c_{1}\left(\mathbf{u}, C, \theta_{1}+\theta_{2}\right) \leq-\mu_{0}\|\mathbf{u}\|_{1}^{2} .
$$

Let input data for problem (75) be such that

$$
4\left(\mathcal{R} e+\mathcal{R} e^{0}\right)\left[1+\left(\gamma_{1} / \gamma_{0}\right) \mathcal{P} \mathcal{R} a\right]<1 .
$$

It follows from (68) under $\gamma_{3}=1$ and (83) that $\mathbf{u}=\mathbf{0}$, and from (57), (47) and relation $\left.\mathbf{u}\right|_{\Gamma}=\mathbf{g}$ we deduce that $C_{1}=C_{2}, p_{1}=p_{2}, \mathbf{g}_{1}=\mathbf{g}_{2}$. So the next theorem holds.

Theorem 9. Let under conditions (i), (ii), ( $j$ ) and (31) $\mathbf{v}_{d} \in \mathbf{H}^{1}(\Omega)$ be a given function, $\mu_{0}>0, \mu_{1} \geq 0$ and the condition (84) takes place where $\mathcal{R} e^{0}=\left(\gamma_{0} / \delta_{0} v\right)\left\|\mathbf{v}_{d}\right\|_{1}$. Then the solution $(\hat{\mathbf{u}}, \hat{p}, \hat{C}, \hat{\mathbf{g}})$ to problem (75) under $Q=\Omega$ is unique.

Let us consider the one-parameter control problem

$$
J(\mathbf{x}, \mathbf{g}) \equiv \frac{\mu_{0}}{2}\left\|\operatorname{rot} \mathbf{v}-\eta_{d}\right\|_{Q}^{2}+\frac{\mu_{1}}{2}\|\mathbf{g}\|_{1 / 2, \Gamma}^{2} \rightarrow \inf , F(\mathbf{x}, \mathbf{g})=0, \mathbf{x}=(\mathbf{v}, g, S) \in X, \mathbf{g} \in K_{1},
$$

corresponding to the cost functional $I_{3}(\mathbf{v})=\left\|\operatorname{rot} \mathbf{v}-\eta_{d}\right\|_{Q}^{2}$. Denoting by $\left(\mathbf{x}_{i}, u_{i}\right)=$ $\left(\mathbf{u}_{i}, p_{i}, C_{i}, \mathbf{g}_{i}\right), i=1,2$, the solution to problem (85) corresponding to the function $\eta_{d}=\zeta_{d}^{(i)} \in$ $\mathbf{L}^{2}(Q), i=1,2$, and setting $\mathbf{g}=\mathbf{g}_{1}-\mathbf{g}_{2}, \zeta_{d}=\zeta_{d}^{(1)}-\zeta_{d}^{(2)}$ in addition to (34) we note that

$$
\begin{gathered}
\left\langle\left(I_{3}\right)_{\mathbf{u}}^{\prime}\left(\mathbf{x}_{i}\right), \mathbf{w}\right\rangle=2\left(\operatorname{rot} \mathbf{u}_{i}-\zeta_{d}^{(i)}, \operatorname{rot} \mathbf{w}\right)_{Q},\left\langle\left(I_{3}\right)_{\mathbf{u}}^{\prime}\left(\mathbf{x}_{1}\right)-\left(I_{3}\right)_{\mathbf{u}}^{\prime}\left(\mathbf{x}_{2}\right), \mathbf{u}\right\rangle= \\
=2\left(\operatorname{rot} \mathbf{u}-\zeta_{d}, \operatorname{rot} \mathbf{u}\right)_{Q},\left(I_{3}\right)_{C}^{\prime}=0 .
\end{gathered}
$$

In view of (86) relations (35), (37), (51), (53), (62) and estimates (57), (59), (60) do not change while (43) and (32) under $\mathbf{w}=\xi_{i}$ transform to

$$
\begin{gathered}
c\left(\mathbf{u}, \mathbf{u}, \xi_{1}+\xi_{2}\right)+\varkappa c_{1}\left(\mathbf{u}, C, \theta_{1}+\theta_{2}\right)+\mu_{0}\left(\|\operatorname{rot} \mathbf{u}\|_{Q}^{2}-\left(\zeta_{d}, \operatorname{rot} \mathbf{u}\right)_{Q}\right) \leq-\mu_{1}\|\mathbf{g}\|_{1 / 2, \Gamma}^{2} \\
v a_{0}\left(\xi_{i}, \xi_{i}\right)=-c\left(\xi_{i}, \mathbf{u}_{i}, \xi_{i}\right)-\varkappa c_{1}\left(\xi_{i}, C_{i}, \theta_{i}\right)-\mu_{0}\left(\operatorname{rot} \mathbf{u}_{i}-\zeta_{d}^{(i)}, \operatorname{rot} \xi_{i}\right)_{Q}, \quad \xi_{i} \in \mathbf{V} .
\end{gathered}
$$

Using (7) and (29) we have

$$
\left|\left(\operatorname{rot} \mathbf{u}_{i}-\zeta_{d}^{(i)}, \operatorname{rot} \xi_{i}\right)_{Q}\right| \leq\left(\left\|\operatorname{rot} \mathbf{u}_{i}\right\|_{Q}+\left\|\zeta_{d}^{(i)}\right\|_{Q}\right)\left\|\operatorname{rot} \xi_{i}\right\|_{Q} \leq c_{r}\left(c_{r} M_{\mathbf{u}}^{0}+\left\|\zeta_{d}^{(i)}\right\|_{Q}\right)\left\|\xi_{i}\right\|_{1} .
$$

Taking into account (63), (64), (89) we deduce from (88) and (62) that

$$
\left\|\theta_{i}\right\|_{1} \leq \frac{\beta_{1}\left\|\xi_{i}\right\|_{1}}{\delta_{1} \lambda \varkappa},\left(\delta_{0} v-\gamma_{0} M_{\mathbf{u}}^{0}-\frac{\beta_{1} \gamma_{1}}{\delta_{1} \lambda} M_{C}^{0}\right)\left\|\xi_{i}\right\|_{1}^{2} \leq \mu_{0} c_{r}\left(c_{r} M_{\mathbf{u}}^{0}+\left\|\zeta_{d}^{(i)}\right\|_{Q}\right)\left\|\xi_{i}\right\|_{1} .
$$


In view of (45) we obtain from this inequality that $\left\|\xi_{i}\right\|_{1} \leq\left(2 \mu_{0} / \delta_{0} v\right) c_{r}^{2}\left(M_{\mathbf{u}}^{0}+c_{r}^{-1}\left\|\zeta_{d}^{(i)}\right\|_{Q}\right)$ which yields (66), (68) and (69) where

$$
\gamma_{3}=c_{r}^{2}, \mathcal{R} e^{0}=\frac{\gamma_{0}}{\delta_{0} v c_{r}} \max \left(\left\|\zeta_{d}^{(1)}\right\|_{Q},\left\|\zeta_{d}^{(2)}\right\|_{Q}\right)
$$

Let us assume that the condition (70) takes place where $\gamma_{3}$ and $\mathcal{R} e^{0}$ are defined in (90). Using (70) we deduce (71). Taking into account (71) we obtain from (87) that

$$
\mu_{0}\left(\|\operatorname{rot} \mathbf{u}\|_{Q}^{2}-\left(\operatorname{rot} \mathbf{u}, \zeta_{d}\right)_{Q}\right) \leq-\varepsilon \mu_{1}\|\mathbf{g}\|_{1 / 2, \Gamma}^{2}
$$

It follows from (91) that $\|\operatorname{rot} \mathbf{u}\|_{Q}^{2} \leq\left(\operatorname{rot} \mathbf{u}, \zeta_{d}\right)_{Q}$ which yields $\|\operatorname{rot} \mathbf{u}\|_{Q} \leq\left\|\zeta_{d}\right\|_{Q}$ or

$$
\left\|\operatorname{rot} \mathbf{u}_{1}-\operatorname{rot} \mathbf{u}_{2}\right\|_{Q} \leq\left\|\zeta_{d}^{(1)}-\zeta_{d}^{(2)}\right\|_{Q}
$$

Rewriting (91) by (92) in the form $\varepsilon \mu_{1}\|\mathbf{g}\|_{1 / 2, \Gamma}^{2} \leq \mu_{0}\left\|\zeta_{d}\right\|_{Q}^{2}$ and using (59), (60) we obtain the following stability estimates:

$$
\begin{gathered}
\left\|\mathbf{g}_{1}-\mathbf{g}_{2}\right\|_{1 / 2, \Gamma} \leq \sqrt{\frac{\mu_{0}}{\varepsilon \mu_{1}}}\left\|\zeta_{d}^{(1)}-\zeta_{d}^{(2)}\right\|_{Q},\left\|\mathbf{u}_{1}-\mathbf{u}_{2}\right\|_{1} \leq \frac{c_{0}(2 \mathcal{R}+1)}{1-2 \mathcal{R} a} \sqrt{\frac{\mu_{0}}{\varepsilon \mu_{1}}}\left\|\zeta_{d}^{(1)}-\zeta_{d}^{(2)}\right\|_{Q}, \\
\left\|C_{1}-C_{2}\right\|_{1} \leq \frac{\gamma_{1} M_{C}^{0}}{\delta_{1} \lambda} \frac{c_{0}(2 \mathcal{R}+1)}{1-2 \mathcal{R} a} \sqrt{\frac{\mu_{0}}{\varepsilon \mu_{1}}}\left\|\zeta_{d}^{(1)}-\zeta_{d}^{(2)}\right\|_{Q} \\
\left\|p_{1}-p_{2}\right\| \leq \frac{\delta_{0} v c_{0}(2 \mathcal{R}+1)(\mathcal{R}+\mathcal{R} a)}{\beta_{0}(1-2 \mathcal{R} a)} \sqrt{\frac{\mu_{0}}{\varepsilon \mu_{1}}}\left\|\zeta_{d}^{(1)}-\zeta_{d}^{(2)}\right\|_{Q} .
\end{gathered}
$$

Thus we have proved the following theorem.

Theorem 10. Let under conditions (i), (ii), (j) and (31) the quadruple $\left(\mathbf{u}_{i}, p_{i}, C_{i}, \mathbf{g}_{i}\right)$ be a solution to problem (85) corresponding to a given function $\zeta_{d}^{(i)} \in \mathbf{L}^{2}(Q), i=1,2$, and condition (70) holds where $\gamma_{3}$ and $\mathcal{R} e^{0}$ are defined in (90). Then stability estimates (92) and (93) hold true.

We can not prove the stability of the solution to problem (85) in the case where $\mu_{1}=0$. But we can establish the local uniqueness of the solution to problem (85) under more strict conditions on $\Omega$ and boundary vector $\mathbf{g}$ if we replace condition (j) by the next condition:

$\left(\mathrm{j}^{\prime}\right) \Omega$ is a simply connected domain with the boundary $\Gamma \in C^{1,1} ; K_{1} \subset \tilde{\mathbf{H}}^{1 / 2}(\Gamma)$ is a convex closed set consisting of functions $\mathbf{g}$ which satisfy the condition $\left.\mathbf{g} \cdot \mathbf{n}\right|_{\Gamma}=q$ where $q \in H^{1 / 2}(\Gamma)$ is a given function.

Indeed, let us note that (87) takes under $\mu_{1}=0, \zeta_{d}^{(1)}=\zeta_{d}^{(2)}, Q=\Omega$ a form

$$
c\left(\mathbf{u}, \mathbf{u}, \xi_{1}+\xi_{2}\right)+\varkappa c_{2}\left(\mathbf{u}, C, \theta_{1}+\theta_{2}\right) \leq-\mu_{0}\|\operatorname{rot} \mathbf{u}\|^{2} .
$$

Under the first condition in $\left(j^{\prime}\right)$ the difference $\mathbf{g}=\mathbf{g}_{1}-\mathbf{g}_{2}$ has the zero normal component on $\Gamma$. Therefore taking into account the simple connectedness of the domain $\Omega$ we have the estimate $\|\mathbf{u}\|_{1} \leq c_{3}\|\operatorname{rot} \mathbf{u}\|$ with the constant $c_{3}$ depending on $\Omega$ (Girault \& Raviart, 1986). Using this estimate we deduce from (68) where $\gamma_{3}$ and $\mathcal{R} e^{0}$ are given in (90) that

$$
\left|c\left(\mathbf{u}, \mathbf{u}, \xi_{1}+\xi_{2}\right)+\varkappa c_{1}\left(\mathbf{u}, C, \theta_{1}+\theta_{2}\right)\right| \leq 4 \mu_{0} c_{r}^{2} c_{3}^{2}\left(\mathcal{R} e+\mathcal{R} e^{0}\right)\left[1+\left(\gamma_{1} / \gamma_{0}\right) \mathcal{P} \mathcal{R} a\right]\|\operatorname{rot} \mathbf{u}\|^{2} .
$$

Let input data for problem (85) be such that

$$
4 c_{r}^{2} c_{3}^{2}\left(\mathcal{R} e+\mathcal{R} e^{0}\right)\left[1+\left(\gamma_{1} / \gamma_{0}\right) \mathcal{P} \mathcal{R} a\right]<1 .
$$


It follows from (94) and (95) that $\operatorname{rot} \mathbf{u}=\mathbf{0}$ which yields $\mathbf{u}=\mathbf{0}$ or $\mathbf{u}_{1}=\mathbf{u}_{2}$. From (57), (47) and the condition $\left.\mathbf{u}\right|_{\Gamma}=\mathbf{g}$ we deduce that $C_{1}=C_{2}, p_{1}=p_{2}, \mathbf{g}_{1}=\mathbf{g}_{2}$. Thus we have proved the following theorem.

Theorem 11. Let under conditions (i), (ii), ( $\left.j^{\prime}\right)$ and (31) $\eta_{d} \in \mathbf{L}^{2}(\Omega)$ be a given function, $\mu_{0}>0$, $\mu_{1} \geq 0$ and the condition (96) takes place where $\mathcal{R} e^{0}=\left(\gamma_{0} / \delta_{0} v c_{r}\right)\left\|\eta_{d}\right\|$. Then the solution $(\hat{\mathbf{u}}, \hat{p}, \hat{C}, \hat{\mathbf{g}})$ to problem (85) under $Q=\Omega$ is unique.

In conclusion we consider two-parameter control problem corresponding to the cost functional $I_{1}$, i.e. we consider the control problem

$$
\begin{gathered}
J(\mathbf{x}, u) \equiv \frac{\mu_{0}}{2}\left\|\mathbf{v}-\mathbf{v}_{d}\right\|_{Q}^{2}+\frac{\mu_{1}}{2}\|\mathbf{g}\|_{1 / 2, \Gamma}^{2}+\frac{\mu_{2}}{2}\|\chi\|_{\Gamma_{N}}^{2} \rightarrow \text { inf, } F(\mathbf{x}, u)=0, \\
\mathbf{x}=(\mathbf{v}, q, S) \in X, u=(\mathbf{g}, \chi) \in K_{1} \times K_{2} .
\end{gathered}
$$

Let $\left(\mathbf{x}_{1}, u_{1}\right) \equiv\left(\mathbf{u}_{1}, p_{1}, C_{1}, \mathbf{g}_{1}, \chi_{1}\right)$ be a solution to problem (97) which corresponds to a function $\mathbf{v}_{d} \equiv \mathbf{u}_{d}^{(1)} \in \mathbf{L}^{2}(Q),\left(\mathbf{x}_{2}, u_{2}\right) \equiv\left(\mathbf{u}_{2}, p_{2}, C_{2}, \mathbf{g}_{2}, \chi_{2}\right)$ be a solution to problem (97) which corresponds to another function $\tilde{\mathbf{v}}_{d} \equiv \mathbf{u}_{d}^{(2)} \in \mathbf{L}^{2}(Q)$. Setting $\mathbf{g}=\mathbf{g}_{1}-\mathbf{g}_{2}, \mathbf{u}_{d}=\mathbf{u}_{d}^{(1)}-\mathbf{u}_{d}^{(2)}$ in addition to (34), we note that the relations (50) hold true for problem (97). In view of (50) relations (52), (53), (61), (62) and estimates (66), (67) do not change while (43) takes the form

$$
c\left(\mathbf{u}, \mathbf{u}, \xi_{1}+\xi_{2}\right)+\varkappa c_{1}\left(\mathbf{u}, C, \eta_{1}+\eta_{2}\right)+\mu_{0}\left(\|\mathbf{u}\|_{Q}^{2}-\left(\mathbf{u}, \mathbf{u}_{d}\right)_{Q}\right) \leq-\mu_{1}\|\mathbf{g}\|_{1 / 2, \Gamma}^{2}-\mu_{2}\|\chi\|_{\Gamma_{N}}^{2} .
$$

Moreover instead of (51) we have to use the original identity (36). It follows from (37) that $C \in \mathcal{T}$. Setting $S=C$ in (36) and using (9) we obtain that

$$
\lambda a_{1}(C, C)+(k C, C)=-c_{1}\left(\mathbf{u}, C_{1}, C\right)+(\chi, C)_{\Gamma_{N}} .
$$

Using (11), (15) and the first estimate in (56) we deduce from (99) that $\delta_{1} \lambda\|C\|^{2} \leq$ $\gamma_{1} M_{C}^{0}\|\mathbf{u}\|_{1}\|C\|_{1}+\gamma_{2}\|\chi\|_{\Gamma_{N}}\|C\|_{1}$. This yields the following estimate for $\|C\|_{1}$ :

$$
\|C\|_{1} \leq \frac{\gamma_{1} M_{C}^{0}}{\delta_{1} \lambda}\|\mathbf{u}\|_{1}+\frac{\gamma_{2}}{\delta_{1} \lambda}\|\chi\|_{\Gamma_{N}} .
$$

Taking into account (46) we obtain from (100) that

$$
\|C\|_{1} \leq \frac{2 \beta_{1}}{\delta_{0} v} \frac{\gamma_{1} M_{C}}{\delta_{1} \lambda}\|C\|_{1}+c_{0}(2 \mathcal{R}+1) \frac{\gamma_{1} M_{C}^{0}}{\delta_{1} \lambda}\|\mathbf{g}\|_{1 / 2, \Gamma}+\frac{\gamma_{2}}{\delta_{1} \lambda}\|\chi\|_{\Gamma_{N}} .
$$

Using (30) and (46), (47) we deduce the following estimates for $C, \mathbf{u}$ and $p$ :

$$
\begin{gathered}
\|C\|_{1} \leq \frac{c_{0}(2 \mathcal{R}+1)}{(1-2 \mathcal{R} a)} \frac{\gamma_{1} M_{C}^{0}\|\mathbf{g}\|_{1 / 2, \Gamma}}{\delta_{1} \lambda}+\frac{\gamma_{2}\|\chi\|_{\Gamma_{N}}}{\delta_{1} \lambda(1-2 \mathcal{R} a)}, \\
\|\mathbf{u}\|_{1} \leq \frac{c_{0}(2 \mathcal{R}+1)\|\mathbf{g}\|_{1 / 2, \Gamma}}{1-2 \mathcal{R} a}+\frac{2 \beta_{1} \gamma_{2}\|\chi\|_{\Gamma_{N}}}{\delta_{0} v \delta_{1} \lambda(1-2 \mathcal{R} a)}, \\
\|p\| \leq \frac{\delta_{0} v c_{0}(2 \mathcal{R}+1)(\mathcal{R}+\mathcal{R} a)\|\mathbf{g}\|_{1 / 2, \Gamma}}{\beta_{0}(1-2 \mathcal{R} a)}+\frac{\beta_{1}(2 \mathcal{R}+1) \gamma_{2}\|\chi\|_{\Gamma_{N}}}{\beta_{0} \delta_{1} \lambda(1-2 \mathcal{R} a)} .
\end{gathered}
$$


It follows from (12), (13), (66) and (102) that

$$
\begin{gathered}
\left|c\left(\mathbf{u}, \mathbf{u}, \xi_{1}+\xi_{2}\right)\right| \leq \gamma_{0}\|\mathbf{u}\|_{1}^{2}\left(\left\|\xi_{1}\right\|_{1}+\left\|\xi_{2}\right\|_{1}\right) \leq 4 \mu_{0} \gamma_{3} \frac{\left(\mathcal{R} e+\mathcal{R} e^{0}\right)}{(1-2 \mathcal{R} a)^{2}}\left[c_{0}(2 \mathcal{R}+1)\|\mathbf{g}\|_{1 / 2, \Gamma}+\right. \\
\left.\frac{2 \beta_{1}}{\delta_{0} v} \frac{\gamma_{2}}{\delta_{1} \lambda}\|\chi\|_{\Gamma_{N}}\right], \varkappa\left|c_{1}\left(\mathbf{u}, C, \theta_{1}+\theta_{2}\right)\right| \leq \varkappa \gamma_{1}\|\mathbf{u}\|_{1}\|C\|_{1}\left(\left\|\theta_{1}\right\|_{1}+\left\|\theta_{2}\right\|_{1}\right) \leq 4 \mu_{0} \gamma_{3} \frac{\gamma_{1}}{\gamma_{0}} \frac{\beta_{1}}{\delta_{1} \lambda} \times \\
\frac{\left(\mathcal{R} e+\mathcal{R} e^{0}\right)}{\gamma_{0}(1-2 \mathcal{R} a)^{2}}\left[c_{0}(2 \mathcal{R}+1)\|\mathbf{g}\|_{1 / 2, \Gamma}+\frac{2 \beta_{1}}{\delta_{0} v} \frac{\gamma_{2}\|\chi\|_{\Gamma_{N}}}{\delta_{1} \lambda}\right]\left[c_{0}(2 \mathcal{R}+1) \frac{\gamma_{1} M_{C}^{0}\|\mathbf{g}\|_{1 / 2, \Gamma}}{\delta_{1} \lambda}+\frac{\gamma_{2}\|\chi\|_{\Gamma_{N}}}{\delta_{1} \lambda}\right] .
\end{gathered}
$$

Here $\gamma_{3}$ and $\mathcal{R} e^{0}$ are defined in (67). From these inequalities and (30) we deduce that

$$
\left|c\left(\mathbf{u}, \mathbf{u}, \xi_{1}+\xi_{2}\right)+\varkappa c_{1}\left(\mathbf{u}, C, \theta_{1}+\theta_{2}\right)\right| \leq \mu_{0}\left(a_{1}\|\mathbf{g}\|_{1 / 2, \Gamma}^{2}+a_{2}\|\chi\|_{\Gamma_{N}}^{2}\right) .
$$

Here constants $a_{1}$ and $a_{2}$ are given by

$$
\begin{aligned}
& a_{1}=\frac{2 \mu_{0} \gamma_{0} \gamma\left(\mathcal{R} e+\mathcal{R} e^{0}\right)}{(1-2 \mathcal{R} a)^{2}} C_{0}^{2}(2 \mathcal{R}+1)^{2}\left[6+\frac{\gamma_{1}}{\gamma_{0}}(5 \mathcal{R} a+1) \mathcal{P}^{2}\right], \\
& a_{2}=\frac{4 \mu_{0} \gamma_{0} \gamma\left(\mathcal{R} e+\mathcal{R} e^{0}\right)}{\left(\delta_{0} v\right)^{2}(1-2 \mathcal{R} a)^{2}}\left(\frac{2 \beta_{1} r \gamma_{2}}{\delta_{1} \lambda}\right)^{2}\left[3+\frac{\gamma_{1}}{\gamma_{0}}(\mathcal{R} a+2) \mathcal{P}^{2}\right] .
\end{aligned}
$$

Let input data for problem (97) and parameters $\mu_{0}, \mu_{1}, \mu_{2}$ be such that

$$
(1-\varepsilon) \mu_{1} \geq \mu_{0} a_{1},(1-\varepsilon) \mu_{2} \geq \mu_{0} a_{2}, \varepsilon=\text { const }>0 .
$$

In view of (105) we deduce from (103) that

$$
\left|c\left(\mathbf{u}, \mathbf{u}, \xi_{1}+\xi_{2}\right)+\varkappa c_{1}\left(\mathbf{u}, C, \theta_{1}+\theta_{2}\right)\right| \leq(1-\varepsilon) \mu_{1}\|\mathbf{g}\|_{1 / 2, \Gamma}^{2}+(1-\varepsilon) \mu_{2}\|\chi\|_{\Gamma_{N}}^{2} .
$$

Combining (98) and (106) we obtain the inequality

$$
\begin{gathered}
\mu_{0}\left(\|\mathbf{u}\|_{Q}^{2}-\left(\mathbf{u}, \mathbf{u}_{d}\right)_{Q}\right) \leq-c\left(\mathbf{u}, \mathbf{u}, \xi_{1}+\xi_{2}\right)-\varkappa c_{1}\left(\mathbf{u}, C, \theta_{1}+\theta_{2}\right)-\mu_{1}\|\mathbf{g}\|_{1 / 2, \Gamma}^{2}-\mu_{2}\|\chi\|_{\Gamma_{N}} \leq \\
-\varepsilon \mu_{1}\|\mathbf{g}\|_{1 / 2, \Gamma}^{2}-\varepsilon \mu_{2}\|\chi\|_{\Gamma_{N}} .
\end{gathered}
$$

Using (107) we deduce (73) which takes place under conditions (105). The uniqueness of the solution to problem (97) follows from this estimate and (107), (102) when $Q=\Omega$.

Rewriting (107) in view of (73) in the form

$$
\begin{aligned}
\varepsilon \mu_{1}\|\mathbf{g}\|_{1 / 2, \Gamma}^{2}+\varepsilon \mu_{2}\|\chi\|_{\Gamma_{N}}^{2} & \leq c\left(\mathbf{u}, \mathbf{u}, \xi_{1}+\xi_{2}\right)+\varkappa c_{1}\left(\mathbf{u}, C, \theta_{1}+\theta_{2}\right)+\mu_{1}\|\mathbf{g}\|_{1 / 2, \Gamma}^{2}+\mu_{2}\|\chi\|_{\Gamma_{N}}^{2} \leq \\
& \leq-\mu_{0}\|\mathbf{u}\|_{Q}^{2}+\mu_{0}\|\mathbf{u}\|_{Q}\left\|\mathbf{u}_{d}\right\|_{Q} \leq \mu_{0}\left\|\mathbf{u}_{d}\right\|_{Q^{\prime}}^{2}
\end{aligned}
$$

and using (102) we obtain the following stability estimates:

$$
\left\|\mathbf{g}_{1}-\mathbf{g}_{2}\right\|_{\Gamma_{N}} \leq \sqrt{\frac{\mu_{0}}{\varepsilon \mu_{1}}}\left\|\mathbf{u}_{d}^{(1)}-\mathbf{u}_{d}^{(2)}\right\|_{Q},\left\|\chi_{1}-\chi_{2}\right\|_{\Gamma_{N}} \leq \sqrt{\frac{\mu_{0}}{\varepsilon \mu_{2}}}\left\|\mathbf{u}_{d}^{(1)}-\mathbf{u}_{d}^{(2)}\right\|_{Q},
$$




$$
\begin{gathered}
\left\|\mathbf{u}_{1}-\mathbf{u}_{2}\right\|_{1} \leq\left[\frac{c_{0}(2 \mathcal{R}+1)}{(1-2 \mathcal{R} a) \sqrt{\mu_{1}}}+\frac{2 \beta_{1} \gamma_{2}}{\delta_{0} v \delta_{1} \lambda(1-2 \mathcal{R} a) \sqrt{\mu_{2}}}\right] \sqrt{\frac{\mu_{0}}{\varepsilon}}\left\|\mathbf{u}_{d}^{(1)}-\mathbf{u}_{d}^{(2)}\right\|_{Q}, \\
\left\|C_{1}-C_{2}\right\|_{1} \leq\left[\frac{c_{0}(2 \mathcal{R}+1)}{(1-2 \mathcal{R} a)} \frac{\gamma_{1} M_{C}^{0}}{\delta_{1} \lambda \sqrt{\mu_{1}}}+\frac{\gamma_{2}}{\delta_{1} \lambda(1-2 \mathcal{R} a) \sqrt{\mu_{2}}}\right] \sqrt{\frac{\mu_{0}}{\varepsilon}}\left\|\mathbf{u}_{d}^{(1)}-\mathbf{u}_{d}^{(2)}\right\|_{Q}, \\
\left\|p_{1}-p_{2}\right\| \leq \frac{(2 \mathcal{R}+1)}{\beta_{0}(1-2 \mathcal{R} a)}\left[\frac{c_{0} \delta_{0} v(\mathcal{R}+\mathcal{R} a)}{\sqrt{\mu_{1}}}+\frac{\beta_{1} \gamma_{2}}{\delta_{1} \lambda \sqrt{\mu_{2}}}\right] \sqrt{\frac{\mu_{0}}{\varepsilon}}\left\|\mathbf{u}_{d}^{(1)}-\mathbf{u}_{d}^{(2)}\right\|_{Q} .
\end{gathered}
$$

So the next theorem holds.

Theorem 12.Let under conditions (i), (ii), (j) and (31) the quintuple $\left(\mathbf{u}_{i}, p_{i}, C_{i}, \mathbf{g}_{i}, \chi_{i}\right)$ be a solution to problem (97) corresponding to a given function $\mathbf{u}_{d}^{(i)} \in \mathbf{L}^{2}(Q), i=1,2$, and conditions (105) hold where parameters $a_{1}$ and $a_{3}$ are given by relations (104) in which $\gamma_{3}$ and $\mathcal{R} e^{0}$ are defined in (67). Then stability estimates (73) and (108) hold true.

In the same manner one can study two-parameter control problems for another cost functionals entering into (22). Consider for example the following control problem:

$$
\begin{gathered}
J(\mathbf{x}, \mathbf{g}, \chi) \equiv \frac{\mu_{0}}{2}\left\|p-p_{d}\right\|_{Q}^{2}+\frac{\mu_{1}}{2}\|\mathbf{g}\|_{1 / 2, \Gamma}^{2}+\frac{\mu_{2}}{2}\|\chi\|_{\Gamma_{N}}^{2} \rightarrow \text { inf, } \\
F(\mathbf{x}, u)=0, \mathbf{x}=(\mathbf{v}, q, S) \in X, u=(\mathbf{g}, \chi) \in K_{1} \times K_{2},
\end{gathered}
$$

corresponding to the functional $I_{4}$. The following theorem holds.

Theorem 13. Let under conditions (i), (ii), (j) and (31) the quintuple $\left(\mathbf{u}_{i}, p_{i}, C_{i}, \mathbf{g}_{i}, \chi_{i}\right)$ be a solution to problem (109) corresponding to a given function $p_{d}^{(i)} \in L^{2}(Q), i=1,2$, and the conditions (105) hold where parameters $a_{1}$ and $a_{3}$ are given by relations

$$
\begin{gathered}
a_{1}=\frac{2 \mu_{0} \gamma_{0}(\mathcal{R}+\mathcal{R} a) \tilde{M}_{p}}{(1-2 \mathcal{R} a)^{2}} C_{0}^{2}(2 \mathcal{R}+1)^{2}\left[6+\frac{\gamma_{1}}{\gamma_{0}}(5 \mathcal{R} a+1) \mathcal{P}^{2}\right], \\
a_{2}=\frac{4 \mu_{0} \gamma_{0}(\mathcal{R}+\mathcal{R} a) \tilde{M}_{p}}{\left(\delta_{0} v\right)^{2}(1-2 \mathcal{R} a)^{2}}\left(\frac{2 \beta_{1} r \gamma_{2}}{\delta_{1} \lambda}\right)^{2}\left[3+\frac{\gamma_{1}}{\gamma_{0}}(\mathcal{R} a+2) \mathcal{P}^{2}\right]
\end{gathered}
$$

in which

Then the estimate

$$
\tilde{M}_{p}=\beta_{0}^{-1}\left[M_{p}^{0}+\max \left(\left\|p_{d}^{(1)}\right\|_{Q},\left\|p_{d}^{(2)}\right\|_{Q}\right)\right] .
$$

and stability estimates

$$
\left\|p_{1}-p_{2}\right\|_{Q} \leq\left\|p_{d}^{(1)}-p_{d}^{(2)}\right\|_{Q}
$$

$$
\begin{gathered}
\left\|\mathbf{g}_{1}-\mathbf{g}_{2}\right\|_{1 / 2, \Gamma} \leq \sqrt{\frac{\mu_{0}}{\varepsilon \mu_{1}}}\left\|p_{d}^{(1)}-p_{d}^{(2)}\right\|_{Q,}\left\|\chi_{1}-\chi_{2}\right\|_{\Gamma_{N}} \leq \sqrt{\frac{\mu_{0}}{\varepsilon \mu_{2}}}\left\|p_{d}^{(1)}-p_{d}^{(2)}\right\|_{Q}, \\
\left\|\mathbf{u}_{1}-\mathbf{u}_{2}\right\|_{1} \leq\left[\frac{c_{0}(2 \mathcal{R}+1)}{(1-2 \mathcal{R} a) \sqrt{\mu_{1}}}+\frac{2 \beta_{1} \gamma_{2}}{\delta_{0} v \delta_{1} \lambda(1-2 \mathcal{R} a) \sqrt{\mu_{2}}}\right] \sqrt{\frac{\mu_{0}}{\varepsilon}}\left\|p_{d}^{(1)}-p_{d}^{(2)}\right\|_{Q}, \\
\left\|C_{1}-C_{2}\right\|_{1} \leq\left[\frac{c_{0}(2 \mathcal{R}+1)}{(1-2 \mathcal{R} a)} \frac{\gamma_{1} M_{T}^{0}}{\delta_{1} \lambda \sqrt{\mu_{1}}}+\frac{\gamma_{2}}{\delta_{1} \lambda(1-2 \mathcal{R} a) \sqrt{\mu_{2}}}\right] \sqrt{\frac{\mu_{0}}{\varepsilon}}\left\|p_{d}^{(1)}-p_{d}^{(2)}\right\|_{Q},
\end{gathered}
$$




$$
\left\|p_{1}-p_{2}\right\| \leq \frac{(2 \mathcal{R}+1)}{\beta_{0}(1-2 \mathcal{R} a)}\left[\frac{c_{0} \delta_{0} v(\mathcal{R}+\mathcal{R} a)}{\sqrt{\mu_{1}}}+\frac{\beta_{1} \gamma_{2}}{\delta_{1} \lambda \sqrt{\mu_{2}}}\right] \sqrt{\frac{\mu_{0}}{\varepsilon}}\left\|p_{d}^{(1)}-p_{d}^{(2)}\right\|_{Q}
$$

hold true.

Similar theorems can be formulated and proved for Models 2 and 3. Details can be found in (Alekseev, 2006; 2007a; Alekseev \& Soboleva, 2009; Alekseev \& Tereshko, 2010a; Alekseev \& Khludnev, 2010).

\section{Numerical algorithm. Results of numerical experiments for Model 2}

In this section we discuss results of computational experiments related to the numerical solution of control problem (4) for Model 2. Our numerical algorithm will be based on optimality system for control problem (4) which is the analogue of relations (25), (26) and (27) for control problem (21). For the sake of simplicity we consider the case where control sets $K_{1}$ and $K_{3}$ coincide with spaces $\tilde{\mathbf{H}}^{1}(\Omega)$ and $L^{2}\left(\Gamma_{N}\right)$ respectively. In this case the minimum is reached in an internal point of set $K$ and it is possible to express optimal controls $\mathbf{g}$ and $\eta$ via adjoint state by explicit formulas (see (Alekseev \& Tereshko, 2010c))

$$
\mathbf{g}=(\sigma \mathbf{n}-v \partial \xi / \partial n) / \mu_{1}, \eta=\theta / \mu_{2} .
$$

Using these expressions we can rewrite the optimality system as a nonlinear operator equation

$$
\Phi(\mathbf{u}, p, T, \xi, \sigma, \theta)=0 .
$$

For its numerical solution the iterative algorithm based on Newton's method is proposed. This algorithm consists of following steps:

1. For given $\left(\mathbf{u}_{0}, p_{0}, T_{0}, \xi_{0}, \sigma_{0}, \theta_{0}\right)$ and supposing $\mathbf{u}_{n}, p_{n}, T_{n}, \xi_{n}, \sigma_{n}, \theta_{n}$ are known, we define $\tilde{\mathbf{u}}$, $\tilde{p}, \tilde{T}, \tilde{\xi}, \tilde{\sigma}, \tilde{\theta}$ by solving the following linear problem:

$$
\Phi^{\prime}\left(\mathbf{u}_{n}, p_{n}, T_{n}, \xi_{n}, \sigma_{n}, \theta_{n}\right)(\tilde{\mathbf{u}}, \tilde{p}, \tilde{T}, \tilde{\xi}, \tilde{\sigma}, \tilde{\theta})=-\Phi\left(\mathbf{u}_{n}, p_{n}, T_{n}, \xi_{n}, \sigma_{n}, \theta_{n}\right) .
$$

2. Then we calculate new approximations $\mathbf{u}_{n+1}, p_{n+1}, T_{n+1}, \xi_{n+1}, \sigma_{n+1}, \theta_{n+1}$ for $\mathbf{u}, p, T, \xi, \sigma$, $\theta$ as

$$
\begin{gathered}
\mathbf{u}_{n+1}=\mathbf{u}_{n}+\tilde{\mathbf{u}}, p_{n+1}=p_{n}+\tilde{p}, T_{n+1}=T_{n}+\tilde{T}, \\
\xi_{n+1}=\xi_{n}+\tilde{\xi}, \sigma_{n+1}=\sigma_{n}+\tilde{\sigma}, \theta_{n+1}=\theta_{n}+\tilde{\theta} .
\end{gathered}
$$

3. If the condition $\left\|T_{n+1}-T_{n}\right\| /\left\|T_{n}\right\|<\varepsilon$ for some sufficiently small number $\varepsilon$ is not satisfied, then we go to step 1.

Below we shall present some numerical tests which illustrate an application of the proposed algorithm to the control problem (4). The first example is connected with the vortex reduction in the steady 2D viscous fluid flow around a cylinder in a channel. As a control we use heat flux $\eta$ on some parts of the boundary. The initial uncontrolled flow is the solution of the boundary-value problem for the dimensionless stationary Navier-Stokes equations

$$
\begin{gathered}
-\frac{1}{\operatorname{Re}} \Delta \mathbf{u}+(\mathbf{u} \cdot \operatorname{grad}) \mathbf{u}+\operatorname{grad} p=\mathbf{0}, \operatorname{div} \mathbf{u}=0 \text { in } \Omega=\Omega_{2} \backslash \Omega_{1}, \\
\left.\mathbf{u}\right|_{\Gamma_{0}}=\mathbf{0},\left.\mathbf{u}\right|_{\Gamma_{1}}=\mathbf{g}_{1}(y),\left.\left(\frac{1}{\operatorname{Re}} \frac{\partial \mathbf{u}}{\partial n}-p \mathbf{n}\right)\right|_{\Gamma_{2}}=\mathbf{0} .
\end{gathered}
$$




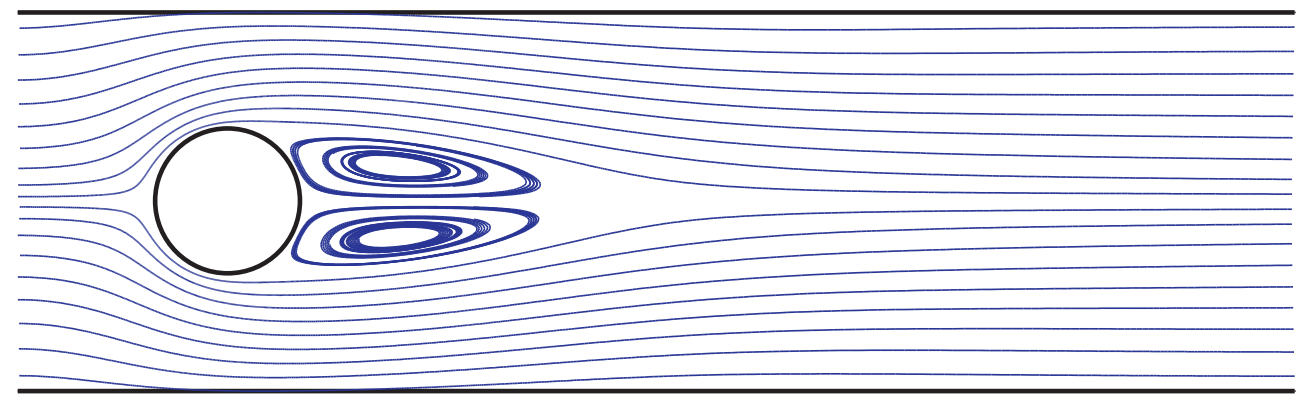

Fig. 1. Streamlines for uncontrolled flow $(\operatorname{Re}=100)$

Here $\operatorname{Re}=U L / v$ is the Reynolds number, $U$ and $L$ are characteristic velocity and length for a flow. We prescribe the no-slip condition on the solid boundaries $\Gamma_{0}$ (the surface of the cylinder and channel walls), a parabolic inflow profile for the velocity on the inlet segment $\Gamma_{1}$ and "do-nothing" boundary conditions on the outlet $\Gamma_{2}$. The streamlines for uncontrolled flow are shown in Fig. 1. They were obtained by solving the boundary-value problem (110) using Newton's method. The open source software freeFEM++ (www.freefem.org) with an adaptive triangular mesh (about 3000 elements) is used for numerical solution of a linearized boundary-value problem. Usually 4 iterations were required for convergence of Newton's method when the solution to the corresponding Stokes problem plays the role of an initial guess.

The flow separation past the body can be clearly identified in Fig. 1. In order to reduce this recirculation the vorticity functional $I_{3}$ with $\zeta_{d}=0$ in (22) is minimized using the proposed algorithm for the dimensionless analogue of Model 2. In this case we need to use additional dimensionless parameter - the Rayleigh number $\mathrm{Ra}=\tilde{\beta} G L^{3} \Delta T /(v \lambda)$, where $\Delta T$ is a characteristic temperature difference. Firstly we choose the heat flux $\eta$ only on the cylinder surface as control. The streamlines of the controlled flow for this case are shown in Fig. 2. One can see that flow separation past the body is eliminated.

In order to obtain a more laminarized flow we expand the area of action of temperature control. In Fig. 3 the controlled flow is presented for the case when the temperature control acts both on the cylinder surface and on the nearest parts of the channel walls (marked with dashed lines in Fig. 3). The vorticity of this flow is small and there is no recirculation zone past the cylinder. An analysis of the temperature field shows that the heat fluxes on additional parts of the boundary compensate a high temperature action on the cylinder surface.

\begin{tabular}{|cc|c|c|c|c|c|}
\hline & & $\mathrm{Re}=20$ & $\mathrm{Re}=40$ & $\mathrm{Re}=60$ & $\mathrm{Re}=80$ & $\mathrm{Re}=100$ \\
\hline Fig. 1 & $\|\operatorname{rot} \mathbf{u}\|$ & 7.68 & 8.47 & 9.14 & 9.71 & 10.22 \\
& $C_{D}$ & 4.25 & 2.77 & 2.26 & 2.01 & 1.85 \\
\hline Fig. 2 & $\|\operatorname{rot} \mathbf{u}\|$ & 7.27 & 7.56 & 7.84 & 8.10 & 8.35 \\
& $C_{D}$ & 4.06 & 2.60 & 2.16 & 1.95 & 1.83 \\
\hline Fig. 3 & $\|\operatorname{rot} \mathbf{u}\|$ & 7.81 & 8.00 & 8.11 & 8.22 & 8.33 \\
& $C_{D}$ & 5.42 & 3.03 & 2.12 & 1.64 & 1.34 \\
\hline
\end{tabular}

Table 1 . Norm of the vorticity and drag coefficient values 


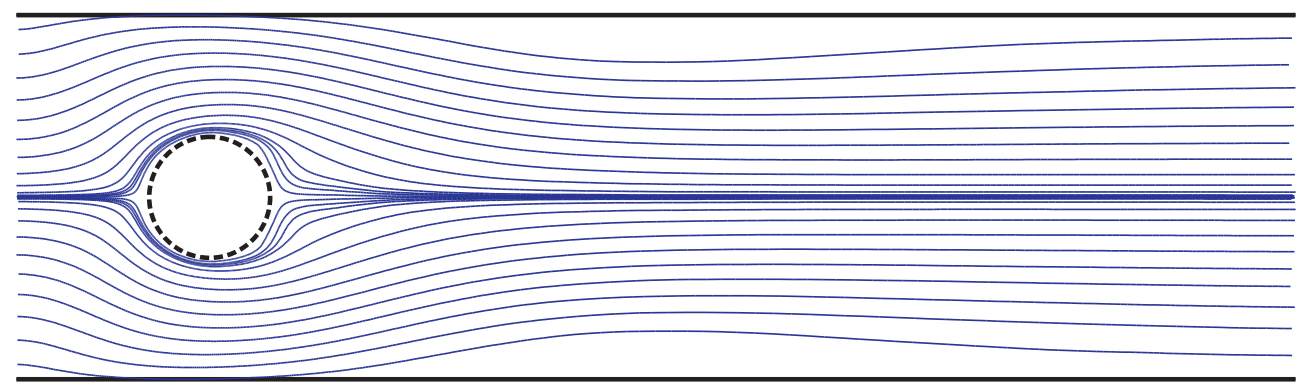

Fig. 2. Streamlines for the heat flux control on the cylinder $(\operatorname{Re}=100)$

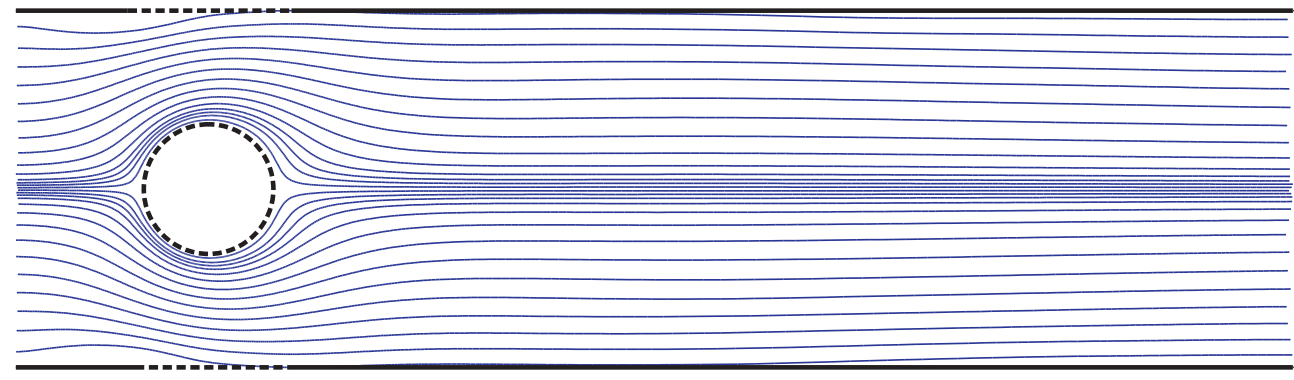

Fig. 3. Streamlines for the heat flux control on the cylinder and on the channel walls $(\operatorname{Re}=100)$

In order to analyze the efficiency of different types of boundary control we calculate the norm of the vorticity $\|\operatorname{rot} \mathbf{u}\|$ and the drag coefficient $C_{D}$ for different values of Reynolds number Re. These values for uncontrolled and controlled flows are shown in Table 1. The smallest values of these parameters correspond to the last case of boundary control.

Analogous results were obtained for the steady 2D viscous fluid flow around a cylinder in a channel with forward-facing step. The streamlines for uncontrolled flow are shown in Fig. 4. One can see a flow separation past the body and vortex at the corner. In order to reduce these recirculations the vorticity functional $I_{3}$ with $\zeta_{d}=0$ in (22) is minimized. In Fig. 5 the controlled flow is presented for the case when the heat flux control acts both on the cylinder surface and on the nearest parts of the channel walls.

The regularization parameter $\mu_{3}$ plays an important role in computations. If $\mu_{3}$ is small then we usually obtain a flow with high temperature gradients on the cylinder surface because a substantial change of the velocity field requires powerful temperature action. This is the reason for the simultaneous use of two controls: $g$ and $\eta$. If main change in the velocity field is achieved due to hydrodynamic control $\mathbf{g}$ then temperature control $\eta$ will play only auxiliary role and temperature gradients will be small.

In the next example we consider two types of control on different parts of the boundary. As in the first example the heat flux on the cylinder surface and on the nearest parts of the channel walls is used to avoid the flow separation past the body. Additionally the Dirichlet control $\mathbf{g}$ on a part $\Gamma_{\mathcal{C}}$ of the boundary $\Gamma$ (marked with dotted lines) is used for corner vortex suppression. Streamlines of corresponding controlled flow are shown in Fig. 6. 


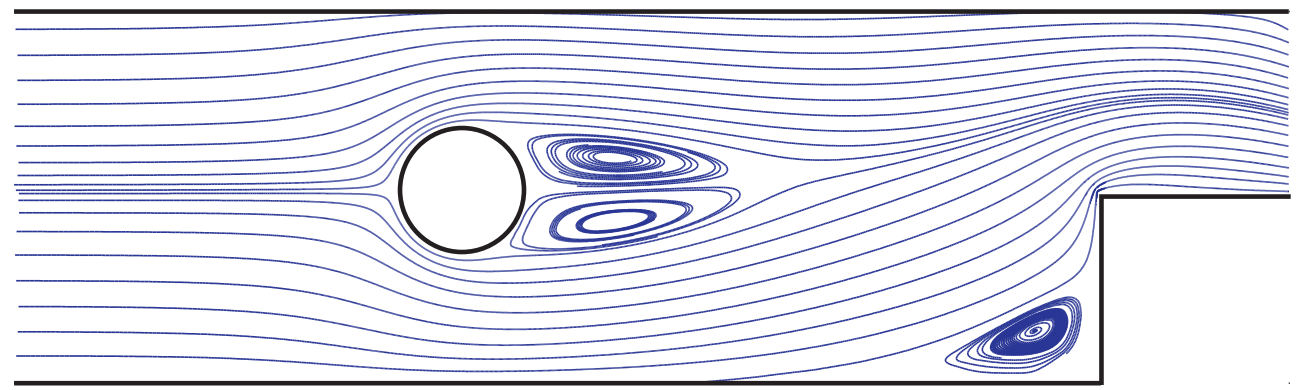

Fig. 4. Streamlines for uncontrolled flow $(\operatorname{Re}=50)$

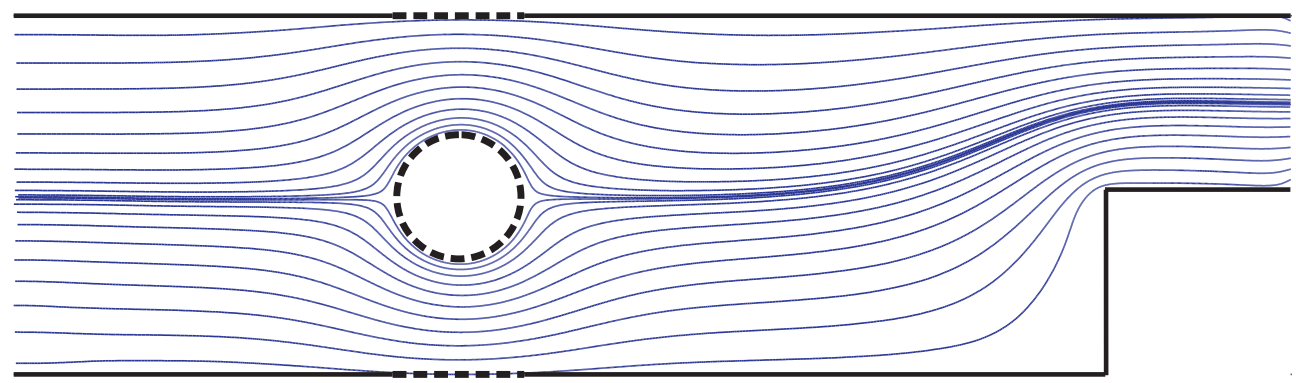

Fig. 5. Streamlines for the heat flux control on the cylinder and on the channel walls $(\operatorname{Re}=50)$

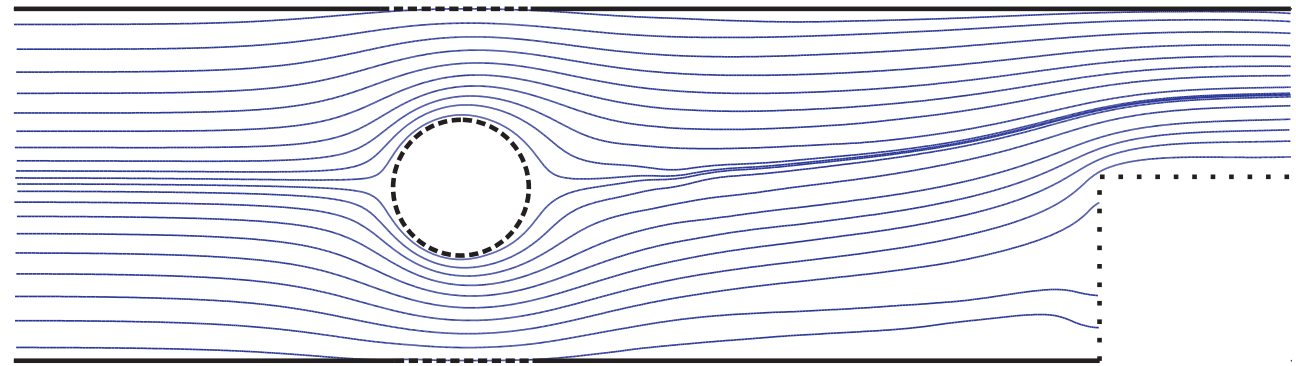

Fig. 6. Streamlines for the heat flux control on the cylinder and on the channel walls and velocity control on the step $(\operatorname{Re}=50)$ 


\begin{tabular}{|cc|c|c|c|c|c|}
\hline & & $\mathrm{Re}=10$ & $\mathrm{Re}=20$ & $\mathrm{Re}=30$ & $\mathrm{Re}=40$ & $\mathrm{Re}=50$ \\
\hline Fig. 4 & $\|\operatorname{rot} \mathbf{u}\|$ & 9.04 & 9.46 & 9.89 & 10.29 & 10.64 \\
& $C_{D}$ & 7.16 & 4.09 & 3.11 & 2.63 & 2.34 \\
\hline Fig. 5 & $\|\operatorname{rot} \mathbf{u}\|$ & 8.88 & 9.04 & 9.22 & 9.41 & 9.60 \\
& $C_{D}$ & 6.21 & 2.98 & 2.02 & 1.59 & 1.34 \\
\hline Fig. 6 & $\|\operatorname{rot} \mathbf{u}\|$ & 8.38 & 8.52 & 8.76 & 8.84 & 9.00 \\
& $C_{D}$ & 6.36 & 3.00 & 2.00 & 1.54 & 1.28 \\
\hline
\end{tabular}

Table 2. Norm of the vorticity and drag coefficient values

The flow separation past the body is eliminated and the corner vortex is absent in this case because of fluid suction at the corner. Calculated values of the norm of the vorticity and of the drag coefficient $C_{D}$ are shown in Table 2 . It can be observed that a simultaneous application of temperature and hydrodynamic controls results in the smallest values of $\|\operatorname{rot} \mathbf{u}\|$. Moreover in this case we obtain the smallest values of the temperature gradients.

The computational experiments showed that if the initial guess is selected sufficiently close to the exact solution, then the algorithm converges for several iterations. The regularization parameter $\mu_{3}$ plays an important role. If its values are relatively large then we can not obtain small values of the functional $I_{3}$. But, on the other hand, the very small values of the regularization parameter can lead to the instability and oscillations in the numerical solution. An analysis of computational results shows that proposed method can be effectively used to produce flows in channels with desired dynamical properties. Some results of other numerical experiments can be found in (Tereshko, 2009; Alekseev \& Tereshko, 2010b;c).

\subsection{Acknowledgments}

This work was supported by the Russian Foundation for Basic Research (projects no. 09-01-98518-r-vostok-a and 10-01-00219-a), Siberian Branch of the Russian Academy of Sciences (project no. 116-2009) and Far Eastern Branch of the Russian Academy of Sciences (projects no. 09-I-P29-01 and 09-I-OMN-03).

\section{References}

Alekseev, G.V. (1998a). Solvability of stationary boundary control problems for heat convection equations. Siberian Mathematical Journal, Vol. 39, No. 5, Sep 1998, 844-858. ISSN 0037-4466

Alekseev, G.V. (1998b). Stationary problems of boundary control for the heat-convection equations. Doklady Akademii Nauk, Vol. 362, No. 2, Sep 1998, 174-177. ISSN 1064-5624

Alekseev, G.V. \& Tereshko, D.A. (1998a). On solvability of inverse extremal problem for stationary equations of viscous heat conducting fluid. Journal of Inverse and Ill-Posed Problems, Vol. 6, No. 6, Jan 1998, 521-562. ISSN 0928-0219

Alekseev, G.V. \& Tereshko, D.A. (1998b). Stationary problems of optimal control for equations of the hydrodynamics of a viscous heat-conducting fluid. Sibirskii Zhurnal Industrial'noi Matematiki, Vol. 1, No. 2, May 1998, 24-44. ISSN 1560-7518 (In Russian)

Alekseev, G.V. (2000). Inverse extremum problems for stationary equations of heat and mass transfer. Doklady Mathematics, Vol. 62, No. 3, Dec 2000, 420-424. ISSN 1064-5624

Alekseev, G.V. \& Adomavichus, E.A. (2001). Theoretical analysis of inverse extremum 
problems of admixture diffusion in viscous fluids. Journal of Inverse and Ill-Posed Problems, Vol. 9, No. 5, Jan 2001, 435-468. ISSN 0928-0219

Alekseev, G.V. (2001). Solvability of inverse extremum problems for stationary equations of heat and mass transfer. Siberian Mathematical Journal, Vol. 42, No. 5, Sep 2001, 811-827. ISSN 0037-4466

Alekseev, G.V. (2002). Inverse extremal problems for stationary equations in mass transfer theory. Computational Mathematics and Mathematical Physics, Vol. 42, No. 3, Sep 2002, 363-376. ISSN 0965-5425

Alekseev, G.V. (2006). Inverse extremum problems for stationary equations of heat convection. Vestnik NGU, Vol. 6, No. 2, Jul 2006, 6-32. ISSN 1818-7994 (In Russian)

Alekseev, G.V. (2007a). Coefficient inverse extremum problems for stationary heat and mass transfer equations. Computational Mathematics and Mathematical Physics, Vol. 47, Feb 2007, 1055-1076. ISSN 0965-5425

Alekseev, G.V. (2007b). Uniqueness and stability in coefficient identification problems for a stationary model of mass transfer. Doklady Mathematics, Vol. 76, No. 2, Feb 2007, 797-800. ISSN 1064-5624

Alekseev, G.V. \& Kalinina, E.A. (2007). Coefficient identification problem for stationary convection-reaction-diffusion equation Sibirskii Zhurnal Industrialnoi Matematiki, Vol. 10, No. 1, Jan 2007, 3-16. ISSN: 1560-7518 (In Russian)

Alekseev, G.V.; Soboleva, O.V. \& Tereshko, D.A. (2008). Identification problems for stationary model of mass transfer. Journal of Applied Mechanics and Technical Physics, Vol. 49, No. 4, Apr 2008, 24-35. ISSN 0021-8944

Alekseev, G.V. \& Tereshko, D.A. (2008). Analysis and optimization in viscous fluid dynamics, Dalnauka, ISBN 978-5-8044-1045-3, Vladivostok (In Russian)

Alekseev, G.V. \& Soboleva, O.V. (2009). On stability of solutions of extremum problems for stationary equations of mass transfer. Dal'nevostochnyi matematicheskii zhurnal, Vol. 9, No. 1-2, Sep 2009, 5-14, ISSN 1608-845X (In Russian)

Alekseev, G.V. \& Khludnev, A.M. (2010). The stability of solutions to extremal problems of boundary control for stationary heat convection equations. Sibirskii Zhurnal Industrial'noi Matematiki, Vol. 13, No. 2, May 2010, 5-18. ISSN 1560-7518 (In Russian)

Alekseev, G.V. \& Tereshko D.A. (2010a). Boundary control problems for stationary equations of heat transfer, In: New Directions in Mathematical Fluid Mechanics, Fursikov, A.V.; Galdi, G.P.; Pukhnachev, V.V. (Eds.), 1-21, Birkhauser Verlag, ISBN 978-3-0346-0151-1, Basel

Alekseev, G.V. \& Tereshko, D.A. (2010b). Extremum problems of boundary control for a stationary thermal convection model. Doklady Mathematics, Vol. 81, No. 1, Feb 2010, 151-155. ISSN 1064-5624

Alekseev, G.V. \& Tereshko, D.A. (2010c). Extremum problems of boundary control for the stationary model of heat convection. Journal of Applied Mechanics and Technical Physics, Vol. 51, No. 4, Jul 2010, 453-463. ISSN 0021-8944

Andreev, V.K.; Kaptsov, O.V.; Pukhnachov, V.V. \& Rodionov, A.A. (1998). Applications of group-theoretical methods in hydrodynamics. Kluwer Academic Publishers, ISBN 978-0-7923-5215-0, Dordrecht

Andreev, V.K.; Gaponenko, Yu.A.; Goncharova, O.A. \& Pukhnachev, V.V. (2008). Modern mathematical models of convection. Fizmatlit, ISBN 978-5-9221-0905-5, Moscow (in Russian)

Batchelor, G.K. (2000). An introduction to fluid dynamics. Cambridge University Press, ISBN 
978-0-5216-6396-0, Cambridge

Capatina, A. \& Stavre, R. (1998). A control problem in bioconvective flow. Journal of Mathematics of Kyoto University, Vol. 37, No. 4, Oct 1998, 585-595. ISSN 0023-608X

Gershuni, G.Z. \& Zhukhovitskii, E.M. (1976). Convective stability of incompressible fluids. Keter, ISBN 978-0-7065-1562-6, Jerusalem

Girault, V., Raviart, P.A. (1986). Finite element methods for Navier-Stokes equations. Theory and algorithms. Springer-Verlag, ISBN 978-0-3871-5796-2, Berlin, New York

Goncharova, O.N. (2002). Unique solvability of a two-dimensional non-stationary problem for the convection equations with temperature depending viscosity. Differential Equations, Vol. 38, No. 2, Feb 2002, 249-258. ISSN 0012-2661

Gunzburger, M.D.; Hou, L. \& Svobodny, T.P. (1991). Analysis and finite element approximation of optimal control problems for the stationary Navier-Stokes equations with distributed and Neumann controls. Mathematics of Computation, Vol. 57, Jul 1991, 123-151. ISSN 0025-5718

Gunzburger, M.D.; Hou, L. \& Svobodny, T.P. (1993). The approximation of boundary control problems for fluid flows with an application to control by heating and cooling. Computers \& Fluids, Vol. 22, Mar 1993, 239-251. ISSN 0045-7930

Hopf, E. (1941). Ein allgemeiner Endlichkeitssatz der Hydrodynamik. Mathematische Annalen, Vol. 117, 1940-1941, 764-775. ISSN 0025-5831

Ito, K. \& Ravindran, S.S. (1998). Optimal control of thermally convected fluid flows. SIAM Journal on Scientific Computing, Vol. 19, No. 6, Nov 1998, 1847-1869. ISSN 1064-8275

Ioffe, A.D. \& Tikhomirov, V.M. (1979). Theory of extremal problems. North Holland, ISBN 978-0-4448-5167-3, Amsterdam

Joseph, D.D. (1976). Stability of fluid motions. Springer-Verlag, ISBN 978-0-4711-1621-9, New York

Lee, H.C. \& Imanuvilov, O.Yu. (2000a). Analysis of optimal control problems for the 2-D stationary Boussinesq equations. Journal of Mathematical Analysis and Applications, Vol. 242, No. 2, Feb 2000, 191-211. ISSN 0022-247X

Lee, H.C. \& Imanuvilov, O.Yu. (2000b). Analysis of Neumann boundary optimal control problems for the stationary Boussinesq equations including solid media. SIAM Journal on Control and Optimization, Vol. 39, No. 2, Sep 2000, 457-477. ISSN 0363-0129

Lee, H.C. (2003). Analysis and computational methods of Dirichlet boundary control problems for 2D Boussinesq equations. Advances in Computational Mathematics, Vol. 19, No. 1-3, Jul 2003, 255-275. ISSN 1019-7168

Perera, P.S. \& Sekerka, R.F. (1997). Nonsolenoidal flow in a liquid diffusion couple. Physics of Fluids. Vol. 9, No. 2, Feb 1997, 376-391. ISSN 1070-6631

Pukhnachov, V.V. (1992). Model of convective flow under low gravity. Microgravity Quarterly, Vol. 2, 1992, 251-252. ISSN 0958-5036

Pukhnachov, V.V. (2004). Hierarchy of models in the theory of convection. Journal of Mathematical Sciences, Vol. 123, No. 6, Oct 2004, 4607-4620. ISSN 1072-3374

Pukhnachev, V.V. (2009). J. Leray problem and V.I. Yudovich conjecture. Proceedings of High Schools. Northern-Caucasian Region. Actual Problems of Mathematical Hydrodynamics, Special Issue, 2009, 185-194 (in Russian)

Pukhnachev, V.V. (2010). Viscous flows in domains with multiply connected boundary, In: Advances in Mathematical Fluid Mechanics, In: New Directions in Mathematical Fluid Mechanics, Fursikov, A.V.; Galdi, G.P.; Pukhnachev, V.V. (Eds.), 333-348, Birkhauser Verlag, ISBN 978-3-0346-0151-1, Basel 
Tereshko, D.A. (2009). Numerical solution of control problems for stationary model of heat convection. Dal'nevostochnyi matematicheskii zhurnal, Vol. 9, No. 1-2, Sep 2009, 168-175, ISSN 1608-845X (In Russian) 


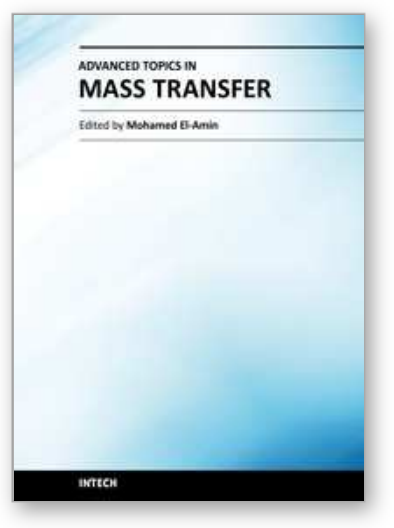

\author{
Advanced Topics in Mass Transfer \\ Edited by Prof. Mohamed El-Amin
}

ISBN 978-953-307-333-0

Hard cover, 626 pages

Publisher InTech

Published online 21, February, 2011

Published in print edition February, 2011

This book introduces a number of selected advanced topics in mass transfer phenomenon and covers its theoretical, numerical, modeling and experimental aspects. The 26 chapters of this book are divided into five parts. The first is devoted to the study of some problems of mass transfer in microchannels, turbulence, waves and plasma, while chapters regarding mass transfer with hydro-, magnetohydro- and electro- dynamics are collected in the second part. The third part deals with mass transfer in food, such as rice, cheese, fruits and vegetables, and the fourth focuses on mass transfer in some large-scale applications such as geomorphologic studies. The last part introduces several issues of combined heat and mass transfer phenomena. The book can be considered as a rich reference for researchers and engineers working in the field of mass transfer and its related topics.

\title{
How to reference
}

In order to correctly reference this scholarly work, feel free to copy and paste the following:

Gennady Alekseev, Dmitry Tereshko and Vladislav Pukhnachev (2011). Boundary Control Problems for Oberbeck-Boussinesq Model of Heat and Mass Transfer, Advanced Topics in Mass Transfer, Prof. Mohamed El-Amin (Ed.), ISBN: 978-953-307-333-0, InTech, Available from: http://www.intechopen.com/books/advancedtopics-in-mass-transfer/boundary-control-problems-for-oberbeck-boussinesq-model-of-heat-and-masstransfer

\section{INTECH}

open science | open minds

\author{
InTech Europe \\ University Campus STeP Ri \\ Slavka Krautzeka 83/A \\ 51000 Rijeka, Croatia \\ Phone: +385 (51) 770447 \\ Fax: +385 (51) 686166 \\ www.intechopen.com
}

\author{
InTech China \\ Unit 405, Office Block, Hotel Equatorial Shanghai \\ No.65, Yan An Road (West), Shanghai, 200040, China \\ 中国上海市延安西路65号上海国际贵都大饭店办公楼 405 单元 \\ Phone: +86-21-62489820 \\ Fax: $+86-21-62489821$
}


(C) 2011 The Author(s). Licensee IntechOpen. This chapter is distributed under the terms of the Creative Commons Attribution-NonCommercialShareAlike-3.0 License, which permits use, distribution and reproduction for non-commercial purposes, provided the original is properly cited and derivative works building on this content are distributed under the same license. 